\title{
Benchmarking atomic data for the CHIANTI atomic database: coronal lines observed by Hinode EIS
}

\author{
G. Del Zanna \\ DAMTP, Centre for Mathematical Sciences, Wilberforce road Cambridge CB3 0WA, UK \\ e-mail: g.del-zanna@damtp.cam.ac.uk
}

Received 28 June 2011 / Accepted 22 August 2011

\begin{abstract}
The coronal lines that are observable in the Hinode EUV Imaging Spectrometer (EIS) wavelengths are assessed with new atomic data that have recently become available for a range of important ions. EIS spectral lines are identified based on their spatial variation across different solar regions, their wavelengths, and their intensities. Two off-limb observations are presented, one above an active region, and one in the quiet Sun. The spectra are relatively free of low-temperature lines. Accurate wavelengths and intensities are provided. The observed and predicted line intensities generally agree excellently, once blending is taken into account. The main diagnostics available for measuring the electron density of the solar corona using EIS are highlighted with the use of the emissivity ratio technique. Densities obtained from different ions agree excellently. Most of the previous identifications are confirmed. A considerable number of weaker lines still await identification, however. The approximate formation temperature of the main unidentified lines is provided to aid the identification process. The EIS radiometric calibration appears to be in need of revision. A new quiet-Sun argon/iron relative abundance of 0.16 , in line with Galactic measurements, is found. The abundances of sulphur and argon in the "background" unresolved active region emission are found to be lower by a factor of about two at $1.5 \mathrm{MK}$, and even lower at $3 \mathrm{MK}$.
\end{abstract}

Key words. atomic data - line: identification - Sun: corona - Sun: abundances - techniques: spectroscopic

\section{Introduction}

This paper is one in a series where atomic data and line identifications are benchmarked against experimental data (Del Zanna et al. 2004, Paper I). A substantial effort was devoted (within the Iron Project (IP) and the UK APAP network) in the past few years to calculate new atomic data for coronal ions and extreme-ultraviolet (EUV) wavelengths, which are important for many solar missions, in particular for the Hinode EUV Imaging Spectrometer (EIS, see Culhane et al. 2007), which covers two wavelength bands (SW: 166-212 A; LW: 245-291 А). New atomic data for Fe viI (Witthoeft \& Badnell 2008), Fe vIII (Del Zanna 2009b), Fe Ix (Storey et al. 2002), Fe x (Del Zanna et al. 2004), Fe xi (Del Zanna et al. 2010), Fe xII (Storey et al. 2005), Fe xIII (Storey \& Zeippen 2010), Fe xIV (Liang et al. 2010), and Si x (Liang et al. 2009) have been produced. These data represent a significant improvement over previous ones. The data for Fe Ix, Fe x, and Fe XII are already available within the CHIANTI database (Dere et al. 1997), and those for Fe vIII, Fe xI, Fe xIII, Fe xIV, and Si x have been included into CHIANTI v.7 (Landi et al. 2011).

These ions are emitting most of the lines in the Hinode/EIS wavelengths. The instrument was radiometrically calibrated on the ground (Lang et al. 2006) with an accuracy of about $20 \%$, hence it is well-suited for benchmarking atomic data. The benchmark for Fe vII, Fe Ix (Del Zanna 2009a), Fe vIII (Del Zanna 2009b), Fe XI (Del Zanna 2010), Fe XIII (Del Zanna 2011) has recently been made also using Hinode/EIS spectra. However, the benchmark of Fex (Del Zanna et al. 2004) and Fe XII (Del Zanna \& Mason 2005) was made with limited experimental data, and many identifications were tentative.
The main aim of this paper is to reassess all previous identifications for the main coronal ions, and suggest which lines are best for diagnostic purposes for use with EIS. The work presented here is a follow-up of the preliminary discussion of the main lines in the EIS spectra given in Young et al. (2007) and is part of the assessment that is routinely made to ensure that the CHIANTI database has the best atomic data.

Brown et al. (2008) provided an extensive list of lines observed with Hinode/EIS, but line identifications were not substantiated with a quantitative analysis. Even at the high spectral resolution of EIS, most lines are blended, and blending strongly depends on the source region. The EUV range is very crowded, and the best way forward is to study clean spectra of different sources. The main hot flare lines have been identified in Del Zanna (2008) and Del Zanna \& Ishikawa (2009). An almost pure EIS spectrum containing only transition-region lines was presented by Del Zanna (2009a). It allowed a detailed benchmark of low-temperature transition-region (TR) emission from a range of cool ions, including Fe vII, Fe VIII and Fe IX. A similar work was independently carried out by Landi \& Young (2009), although the EIS spectrum did contain a mixture of cold and warm (1 MK) emission.

This paper focuses on the coronal lines. To limit contamination from cool lines, off-limb spectra have been carefully selected for this study. This has been done because in on-disk observations of active regions a large number of lines that become visible only at high electron densities appear, and the line identification becomes even more complex. This discussion is left to a separate paper.

The identification and the atomic data for Hinode EIS are of particular relevance for many EUV imagers such as SOHO/EIT 
and the Solar Dynamics Observatory (SDO) Atmospheric Imaging Assembly (AIA), because many of their bands cover the same spectral region (O'Dwyer et al. 2010; Del Zanna et al. 2011). Indeed, the interpretation of imaging data relies on the accuracy of the underlying atomic data. Section 2 outlines the benchmark method. Sections 3 and 4 present off-limb active region and quiet-Sun observations. Section 5 presents a combined summary of the benchmark, while Sect. 6 discusses the relative abundances. Section 7 draws the conclusions.

\section{Benchmark method}

All identifications of the strongest lines were checked with laboratory and solar spectra. Line intensities, whenever available, were compared to confirm identifications and assess the possible presence of blending. For the line identification, we made use of the "emissivity ratio" technique, which divides the observed intensity of a line by its emissivity:

$F_{j i}=\frac{I_{\mathrm{ob}} N_{\mathrm{e}} C}{N_{j}\left(N_{\mathrm{e}}, T_{\mathrm{e}}\right) A_{j i}}$

calculated at a fixed electron temperature $T_{\mathrm{e}}$ (or density $N_{\mathrm{e}}$ ) and plotted as a function of the density $N_{\mathrm{e}}$ (or temperature $T_{\mathrm{e}}$ ). $I_{\mathrm{ob}}$ is the observed intensity in the line. The scaling constant $C$ is chosen so that the curves are close to unity. The same constant is chosen for each plot. If theoretical and observed intensities agree, all $F_{j i}$ curves should either overlap or cross, if the plasma is nearly isodensity and isothermal. This allows one to assess at once, in one single plot, how good observed vs. theoretical intensities are for a group of lines (see Del Zanna et al. 2004 for details).

In the emissivity ratio plots below, the observed wavelength $\lambda_{\mathrm{ob}}(\AA)$ and the indices of the transition(s) contributing to the line are shown. The observed intensity is also shown. Whenever a line is blended with a known line, this is indicated, and the observed intensity decreased by a factor shown in the plot. For example, $95 \%$ of the line at $256.94 \AA$ in the active region observation is caused by the 1-14 transition in Fe XI, and 5\% by the 2-15 transition in FexII. This is indicated in the plots as $\lambda_{\mathrm{ob}} 256.94 \AA(1-14) 0.95 \times I_{\mathrm{ob}}=28.6(\mathrm{Fe} \mathrm{xI})$, and $\lambda_{\mathrm{ob}} 256.94 \AA$ $(2-15) 0.05 \times I_{\mathrm{ob}}=1.5$ (Fe XII), given that the total observed intensity is 30.1 . When a line is a known self-blend of transitions form the same ion, this is indicated as "sbl".

For many lines, the observed intensities are higher than the predicted ones. In these cases, the possible presence of a blend with an unidentified line is listed as "(bl u)", and sometimes the observed intensity is decreased by a factor displayed in the plots to have its emissivity ratio agree with those of the other lines. This provides an indication for the degree to which the observed line appears to be blended. When other effects such as instrument calibration problems could be present, a question mark is shown. New identifications are shown as "N", and tentative new ones as "TN".

Any emission measure modelling is specifically avoided for the benchmark because of the various problems associated with it (Del Zanna et al. 2002). The emissivity ratio method was carried out for all the ions, and the main blending contribution of the various transitions to each observed line was established.

\section{Active region off-limb, 2007 August 19}

Here we consider a long-exposure (90 s) full-spectral observation of an active region at the limb on 2007 August 19. Long
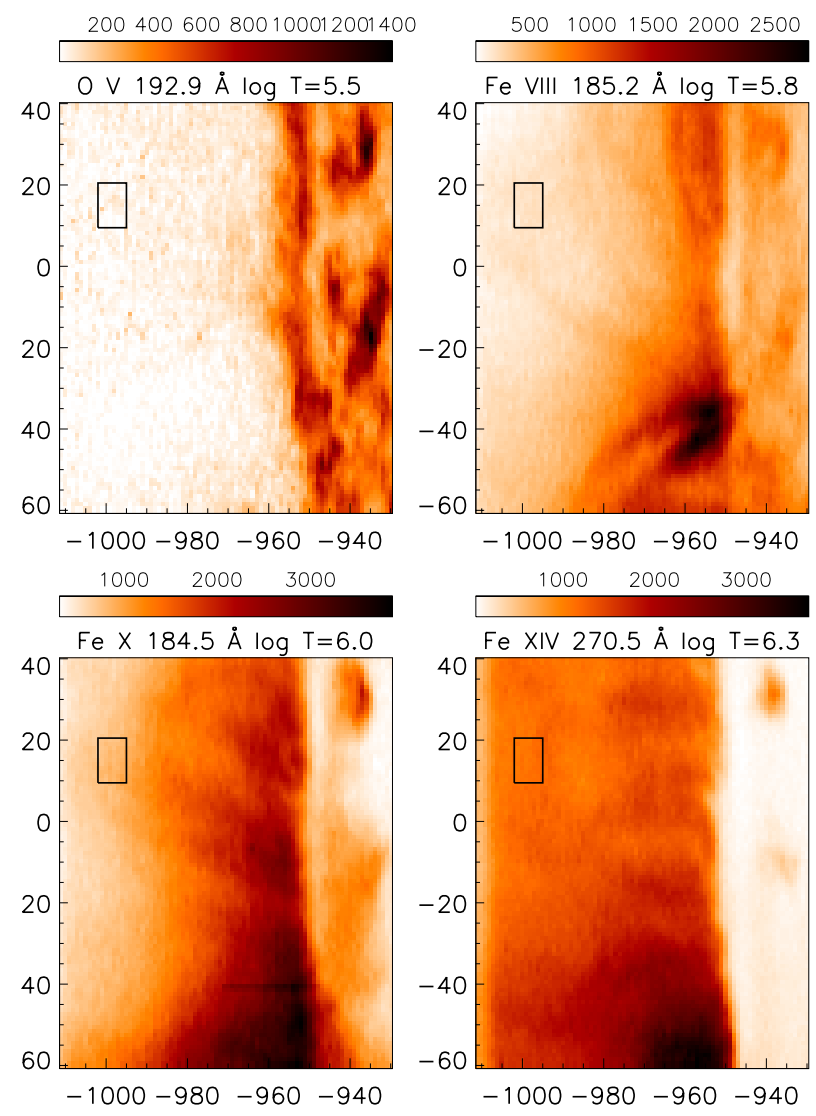

Fig. 1. Monochromatic images (negative, integrated counts) of some of the strongest lines observed by EIS on 2007 Aug. 19 and formed over a range of temperatures. The box represents the area chosen to obtain a sample spectrum. The coordinates are solar $X$ and solar $Y$, in arcseconds from disk centre.

exposures are needed to increase the signal-to-noise, but have the drawback of increasing the incidence of cosmic rays. The data had to be processed with custom-written software by visually inspecting each exposure for cosmic ray hits. A complex data processing, which included various geometrical corrections and a wavelength calibration procedure was applied to the data as described in detail in Del Zanna (2009b). More than 200 lines were fitted with Gaussian profiles using the cfit package (Haugan 1997), and their "morphology" (the aspect of the line radiances) was examined in detail, one by one. Figure 1 shows the resulting monochromatic images for a selection of lines, and the off-limb area chosen to obtain an averaged spectrum, displayed in Fig. 2. This off-limb region is in the unresolved "background" corona outside the core of the active region (which is in the lower part of the field-of-view). This diffuse emission was first described using lower-resolution SOHO/CDS observations by Del Zanna \& Mason (2003) as being pervasive and multi-thermal.

The spectrum was wavelength-calibrated using a selection of coronal lines, assuming that there are no Dopplershifts in the lines, a common and reasonable assumption. The rest wavelengths for EUV coronal ions found in the literature and all databases (e.g. NIST) mostly originate from Behring et al. (1976), who provided a whole-Sun spectrum in the 160$770 \AA$ range with excellent resolution $(0.06 \AA)$. The maximum difference between reference and observed lines was $5 \mathrm{~m} \AA$ for both EIS channels, and in many cases the EIS wavelengths are actually more accurate than the Behring et al. (1976) ones. 
G. Del Zanna: Benchmarking atomic data for the CHIANTI atomic database: coronal lines observed by Hinode EIS
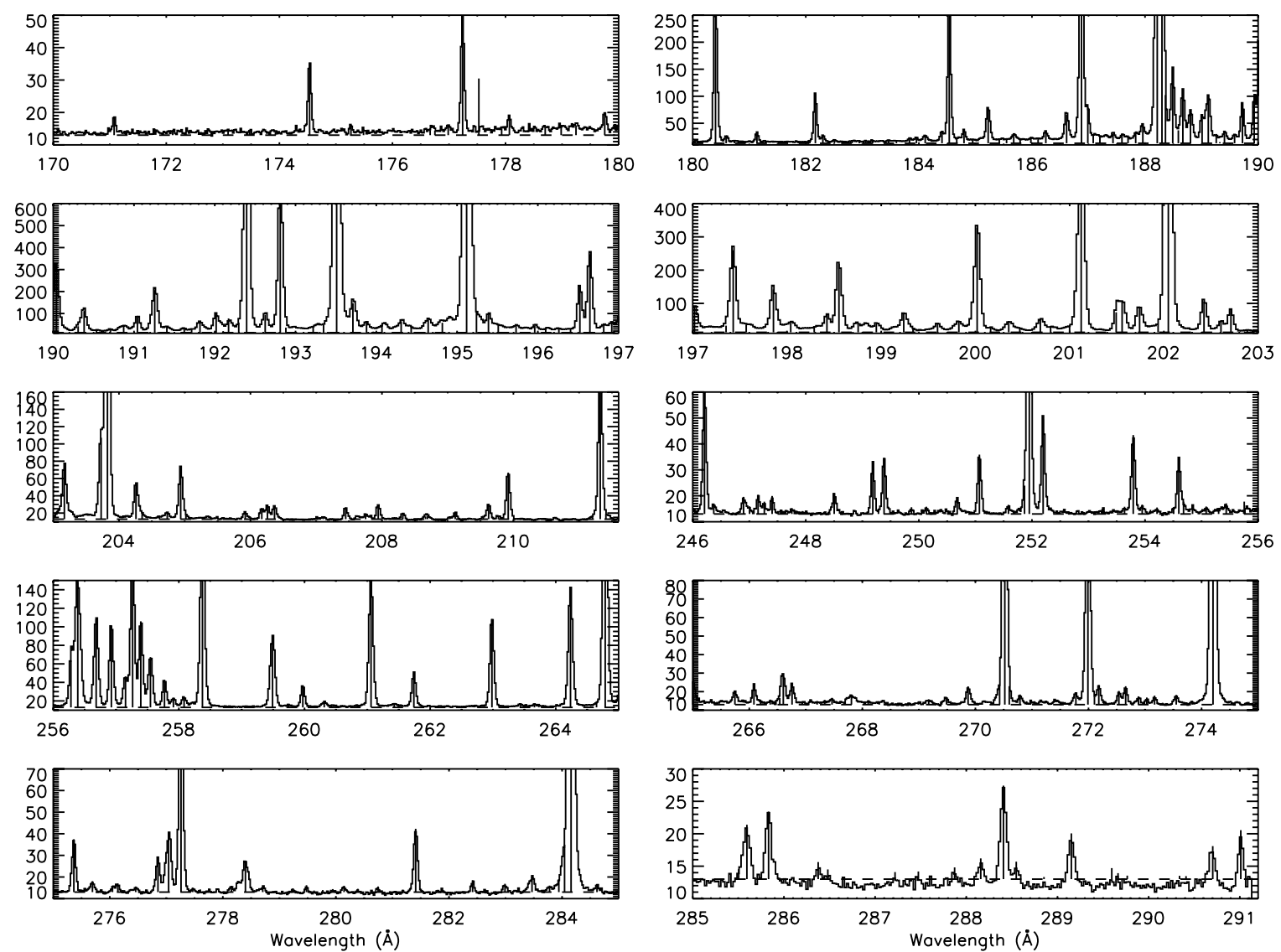

Fig. 2. Hinode EIS off-limb active region spectra recorded on 2007 Aug. 19 (abscissa: wavelengths in $\AA$; ordinate: averaged counts per pixel). The locations of the estimated EIS CCD bias are shown as dashed lines. The locations of all the spectral lines fitted in the spectrum are also shown.

Table 1 provides the list of the strongest lines present in this spectrum, with their measured wavelengths $\lambda_{\mathrm{o}}$ and intensities. Notice that both the intensities in terms of total counts in the lines are given, as well as the calibrated ones. This was done to highlight the fact that many intrinsically weak spectral lines that fall near the peak sensitivity of the channels do indeed have high count rates, so are very well observed. Many wavelengths are within a few $m \AA$ from the literature values, also shown in Table 1. This agreement is remarkable. The table also clearly indicates that a considerable number of rest wavelengths need to be revised.

Figure 3 shows the EM loci curves (see Del Zanna et al. 2002 for an explanation of the method), indicating a multi-thermal plasma, as expected (Del Zanna \& Mason 2003). Strong decays to the ground states have been selected for iron (Fe VIII 185.2, Fex 184.5, FexII 192.4, FexIII 202.0, FexIV 264.8, Fexv 284.2, Fexvi $263 \AA$ ) sulfur (Sx 264.2, S XI 281.4, S XII 288.4, S xIII 256.7 ̊), argon (Ar xI 188.8 and Ar xIV $194 \AA$ ) and calcium (CaxIv 193.9 and Caxv $201 \AA$ ). The curves are displayed with the Asplund et al. (2009) photospheric abundances, with the exception of sulphur, decreased by a factor of 1.7. Nearby off-limb areas show very similar EM loci curves.

\section{Quiet Sun off-limb, 2007 March 11}

Here we consider a long-exposure (90 s) observation on 2007 March 11 of the quiet Sun at the limb, the same as used for the Fe xi benchmark (Del Zanna 2010), although the data were re-processed here with visual inspection for cosmic ray hits. Figure 4 shows the monochromatic images for a selection of

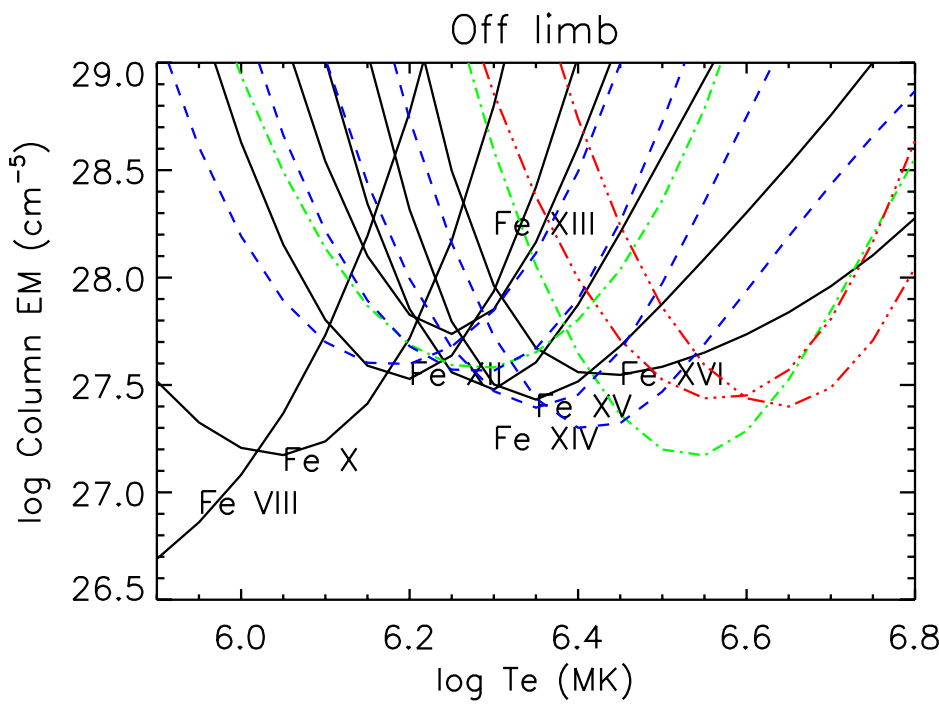

Fig. 3. EM loci curves for the 2007 Aug. 19 off-limb active region spectrum and a selection of iron (solid lines), sulfur (dashed), argon (dot dash), and calcium (dash triple dots) ions. The Asplund et al. (2009) photospheric abundances were adopted (with the exception of sulphur, decreased by a factor of 1.7$)$.

lines to show how they are different for each ion stage. This fact was taken into account for the assessment.

An averaged spectrum was obtained over eight exposures from an off-limb area shown in Fig. 4. This area is farther off-limb compared to the one selected in Del Zanna (2010), 
Table 1. List of emission lines from the 2007 Aug. 19 off-limb active Table 1. continued. region spectrum.

\begin{tabular}{|c|c|c|c|c|c|}
\hline$\underline{\lambda_{0}}$ & DN & Int & $F$ & ID & $\lambda_{\mathrm{r}}$ \\
\hline 171.077 & 19.8 & 265.0 & 77 & Fe IX & 171.072 \\
\hline 174.535 & 77.0 & 172.7 & 72 & $\mathrm{Fe} x$ & 174.534 \\
\hline 175.266 & 11.4 & 20.2 & 77 & $\mathrm{Fe} x$ & 175.263 \\
\hline 175.488 & 6.4 & 10.3 & 77 & $\mathrm{u}$ & \\
\hline 176.751 & 5.0 & 4.7 & 77 & $? \mathrm{Nixv}$ & 176.74 \\
\hline 176.987 & 10.6 & 9.0 & 77 & u (bl oc Fe vII) & 176.950 \\
\hline 177.241 & 126.8 & 95.0 & 72 & $\mathrm{Fe} x$ & 177.242 \\
\hline 178.068 & 21.0 & 11.1 & 76 & Fe $\mathrm{xI}$ & 178.056 \\
\hline 179.247 & 11.0 & 3.6 & 76 & u (? S Ix) & \\
\hline 179.755 & 24.2 & 6.7 & 76 & Fe XI & 179.760 \\
\hline 180.403 & 1006.4 & 221.2 & 71 & Fe $\mathrm{xI}(\mathrm{bl} F \mathrm{x}$ ) & 180.400 \\
\hline 180.591 & 47.5 & 9.8 & 76 & $\mathrm{Fe} \mathrm{xI}$ & 180.595 \\
\hline 180.738 & 16.8 & 3.3 & 76 & $\mathrm{Sx}$ & 180.733 \\
\hline 180.864 & 12.1 & 2.3 & 76 & $\mathrm{u}$ & \\
\hline 181.136 & 70.3 & 12.0 & 76 & Fe xI (bl oc u TR) & 181.131 \\
\hline 182.072 & 15.8 & 1.9 & 76 & u (bl oc Fe vII) & 182.100 \\
\hline 182.169 & 286.7 & 34.3 & 65 & Fe XI & 182.167 \\
\hline 182.301 & 49.5 & 5.7 & 76 & $\mathrm{Fe} x(\mathrm{bl} \mathrm{u})$ & 182.308 \\
\hline 182.507 & 19.8 & 2.1 & 76 & u (? Co XIII) & \\
\hline 182.819 & 14.3 & 1.4 & 76 & & \\
\hline 182.946 & 14.0 & 1.3 & 76 & $\mathrm{u}$ & \\
\hline 183.181 & 20.3 & 1.8 & 79 & $\mathrm{u}$ & \\
\hline 183.464 & 23.0 & 1.8 & 79 & Caxiv (bl u, Ar XIV) & 183.460 \\
\hline 183.603 & 20.2 & 1.6 & 79 & $\mathrm{u}$ & \\
\hline 183.834 & 36.8 & 2.6 & 79 & $\mathrm{u}(\mathrm{bl} \mathrm{oc} \mathrm{Fe} \mathrm{vII)}$ & 183.820 \\
\hline 183.957 & 42.5 & 2.9 & 79 & O vI & 183.937 \\
\hline 184.112 & 59.5 & 3.9 & 79 & O vi (bl Ni VIII?) & 184.117 \\
\hline 184.409 & 87.3 & 5.2 & 79 & u X (bl Fe xI) & \\
\hline 184.538 & 825.7 & 47.9 & 68 & $\mathrm{Fe} x$ & 184.543 \\
\hline 184.670 & 41.4 & 2.3 & 79 & $\mathrm{uX}$ & \\
\hline 184.798 & 92.3 & 5.0 & 79 & Fe xI (bl) & 184.793 \\
\hline 184.952 & 30.1 & 1.6 & 79 & ? Nix & 184.937 \\
\hline 185.225 & 275.3 & 13.4 & 89 & Fe VIII (bl Ni xvI) & \\
\hline 185.423 & 32.5 & 1.5 & 79 & ? Mn VIII & 185.460 \\
\hline 185.551 & 33.7 & 1.5 & 79 & $\mathrm{u}(\mathrm{bl} \mathrm{oc} \mathrm{Fe}$ vII) & \\
\hline 185.678 & 67.3 & 2.9 & 79 & $\mathrm{u}$ & \\
\hline 185.791 & 32.2 & 1.4 & 79 & u (bl Ni xv ?) & \\
\hline 185.981 & 42.1 & 1.7 & 79 & $\mathrm{u}$ & \\
\hline 186.109 & 39.1 & 1.5 & 79 & $\mathrm{u}$ & \\
\hline 186.246 & 87.8 & 3.4 & 79 & Fe XII (bl u) & 186.248 \\
\hline 186.614 & 225.2 & 8.0 & 89 & Fe VIII (bl CaxIv) & 186.61 \\
\hline 186.877 & 1688.7 & 56.7 & 85 & Fe XII (sbl, bl S XI) & 186.880 \\
\hline 186.988 & 208.5 & 6.8 & 65 & Fe XII (bl Ni xI) & 187.000 \\
\hline 187.083 & 62.3 & 2.0 & 79 & $? \mathrm{Mg}$ x (bl Ar xı?) & \\
\hline 187.202 & 50.1 & 1.6 & 79 & $? \mathrm{Mg} \times(\mathrm{bl} \mathrm{Fe} \mathrm{vIII)}$ & 187.18 \\
\hline 187.434 & 64.8 & 1.9 & 79 & $\mathrm{Fe} \mathrm{xI}$ & 187.450 \\
\hline 187.592 & 58.3 & 1.7 & 79 & $\mathrm{u}$ & \\
\hline 187.700 & 42.9 & 1.2 & 79 & $\mathrm{u}$ & \\
\hline 187.835 & 77.3 & 2.2 & 79 & $\mathrm{u}$ & \\
\hline 187.961 & 117.9 & 3.2 & 79 & u VIII-IX (bl Ar xIV) & \\
\hline 188.217 & 4860.7 & 127.3 & 70 & Fe xi (bl oc) & 188.216 \\
\hline 188.300 & 3048.1 & 78.7 & 65 & $\mathrm{Fe} x \mathrm{xI}(\mathrm{bl} \mathrm{u})$ & 188.299 \\
\hline 188.361 & 339.8 & 8.6 & 79 & u XI & \\
\hline 188.490 & 490.6 & 12.3 & 75 & $\mathrm{Fe}$ IX & \\
\hline 188.589 & 80.7 & 2.0 & 70 & u VIII (Ni VIII?) & \\
\hline 188.670 & 361.6 & 8.8 & 75 & S XI (bl Fe xII) & 188.667 \\
\hline 188.805 & 221.4 & 5.3 & 79 & $\operatorname{Ar} \mathrm{XI}$ & 188.82 \\
\hline 189.009 & 188.0 & 4.3 & 79 & Fe XI & 189.020 \\
\hline 189.119 & 338.1 & 7.7 & 79 & Fe xI (bl u) & 189.123 \\
\hline 189.260 & 65.3 & 1.5 & 79 & $\mathrm{u}$ & \\
\hline 189.405 & 81.9 & 1.8 & 79 & u (bl oc Fe vII 189.450) & \\
\hline 189.584 & 59.5 & 1.3 & 79 & $\operatorname{Ar} \mathrm{xI}(\mathrm{bl} \mathrm{u})$ & 189.57 \\
\hline 189.724 & 266.9 & 5.6 & 79 & Fe XI (bl u) & 189.733 \\
\hline 189.931 & 277.7 & 5.6 & 69 & Fe IX & \\
\hline 190.040 & 1144.1 & 22.9 & 79 & Fe $x(b l F e x I I)$ & TN \\
\hline 190.308 & 110.9 & 2.1 & 79 & u XII & \\
\hline 190.382 & 372.8 & 7.1 & 79 & Fe XI (bl u) & 190.390 \\
\hline 190.535 & 39.1 & 0.7 & 79 & $? \mathrm{Mg} \mathrm{x}$ & 190.56 \\
\hline 190.708 & 57.7 & 1.1 & 79 & $\mathrm{u}$ & \\
\hline 190.872 & 109.7 & 2.0 & 79 & $? \mathrm{Nax}$ & 190.874 \\
\hline 191.042 & 266.3 & 4.7 & 79 & Fe XII (bl Ar XI) & 191.050 \\
\hline 191.257 & 733.2 & 12.7 & 79 & S xI (bl Fe Ix) & 191.27 \\
\hline 191.407 & 98.7 & 1.7 & 79 & u (bl Ar XIV) & \\
\hline 191.622 & 72.4 & 1.2 & 79 & ? Mn IX & \\
\hline 191.713 & 41.4 & 0.7 & 65 & $\mathrm{u}$ & \\
\hline 191.817 & 178.1 & 2.9 & 79 & Fe XII (bl) & TN \\
\hline 192.017 & 316.1 & 5.1 & 79 & Fe xI (bl oc many) & 192.01 \\
\hline 192.104 & 23.2 & 0.4 & 69 & u X & \\
\hline 192.171 & 245.5 & 3.9 & 79 & Fe XII & TN \\
\hline 192.297 & 146.3 & 2.3 & 66 & u X & \\
\hline 192.394 & 6068.7 & 94.1 & 70 & Fe XII & 192.390 \\
\hline
\end{tabular}

\begin{tabular}{|c|c|c|c|c|c|}
\hline$\lambda_{\mathrm{o}}$ & DN & Int & $F$ & ID & $\lambda_{\mathrm{r}}$ \\
\hline 192.624 & 305.7 & 4.6 & 79 & $\mathrm{Fe}$ (xi (bl Ni x ?) & 192.630 \\
\hline 192.811 & 2266.0 & 34.0 & 70 & Fe xI (bl Ca XVII ?) & 192.813 \\
\hline 192.880 & 87.0 & 1.3 & 79 & $\mathrm{Ov}$ & 192.910 \\
\hline 193.218 & 93.6 & 1.4 & 79 & $\mathrm{u}$ & \\
\hline 193.347 & 132.4 & 1.9 & 79 & u VII-VIII & \\
\hline 193.509 & 14407.5 & 205.5 & 71 & Fe XII & 193.509 \\
\hline 193.710 & 508.2 & 7.2 & 79 & $\mathrm{Fe} \times(\mathrm{bl} \mathrm{u})$ & 193.715 \\
\hline 193.879 & 186.7 & 2.6 & 79 & Caxiv & 193.866 \\
\hline 193.963 & 14.7 & 0.2 & 65 & Fe vIII & 193.968 \\
\hline 194.070 & 147.0 & 2.0 & 79 & Ar XI & 194.09 \\
\hline 194.308 & 196.8 & 2.7 & 79 & u IX-X (?Mn x) & \\
\hline 194.431 & 93.7 & 1.3 & 79 & Ar xIV & 194.390 \\
\hline 194.642 & 217.5 & 2.9 & 79 & Fe vIII & 194.660 \\
\hline 194.817 & 170.7 & 2.3 & 79 & $\mathrm{u}$ & \\
\hline 195.118 & 23503.1 & 312.1 & 70 & Fe XII (sbl) & 195.119 \\
\hline 195.240 & 371.1 & 4.9 & 79 & u XI-XII (bl Ni xvI) & \\
\hline 195.388 & 328.6 & 4.3 & 79 & u X & \\
\hline 195.510 & 144.3 & 1.9 & 79 & ? Nixv & 195.540 \\
\hline 195.731 & 105.0 & 1.4 & 79 & $\mathrm{u}$ & \\
\hline 195.961 & 119.5 & 1.6 & 79 & Fe vIII & 195.972 \\
\hline 196.098 & 71.2 & 0.9 & 79 & u (bl Fe viI 196.046) & \\
\hline 196.261 & 63.3 & 0.8 & 79 & u (bl Fe vII ) & \\
\hline 196.405 & 69.8 & 0.9 & 79 & $\mathrm{u}$ & \\
\hline 196.520 & 703.6 & 9.4 & 68 & Fe XIII & 196.525 \\
\hline 196.645 & 1261.1 & 17.0 & 74 & Fe xII (bl oc Fe vIII) & 196.640 \\
\hline 196.816 & 139.0 & 1.9 & 79 & u (bl S x 196.814) & \\
\hline 196.915 & 142.1 & 1.9 & 65 & $\mathrm{u}$ & \\
\hline 197.019 & 291.9 & 4.0 & 79 & u XIV & \\
\hline 197.354 & 66.9 & 0.9 & 70 & u (bl Fe VIII) & \\
\hline 197.429 & 840.3 & 11.9 & 71 & Fe XIII (bl Nix?) & 197.433 \\
\hline 197.567 & 89.3 & 1.3 & 79 & ? Nix & \\
\hline 197.857 & 491.7 & 7.3 & 79 & Fe IX & \\
\hline 198.040 & 108.7 & 1.7 & 79 & $\mathrm{u}$ & \\
\hline 198.289 & 53.7 & 0.8 & 79 & u (bl Ni xvI) & \\
\hline 198.424 & 195.8 & 3.2 & 74 & u XI (bl Ni xI) & \\
\hline 198.552 & 773.7 & 12.8 & 78 & S VIII (bl Fe xI) & 198.550 \\
\hline 198.756 & 89.6 & 1.6 & 79 & $\mathrm{u}$ & \\
\hline 198.948 & 93.1 & 1.7 & 79 & $\mathrm{u}$ & \\
\hline 199.239 & 207.4 & 4.0 & 79 & $\mathrm{u}$ & \\
\hline 199.338 & 36.1 & 0.7 & 79 & $\mathrm{u}$ & \\
\hline 199.598 & 92.5 & 1.9 & 79 & $\mathrm{u}$ & \\
\hline 199.824 & 113.0 & 2.6 & 79 & u XI (bl oc Ni XVII) & \\
\hline 200.018 & 1178.6 & 28.0 & 78 & Fe XIII & 200.021 \\
\hline 200.161 & 56.1 & 1.4 & 79 & $\mathrm{u}$ & \\
\hline 200.354 & 120.6 & 3.2 & 79 & u XI-XII & \\
\hline 200.471 & 44.3 & 1.2 & 79 & $\mathrm{u}$ & \\
\hline 200.689 & 149.3 & 4.4 & 79 & u X-XII & \\
\hline 200.798 & 55.7 & 1.7 & 79 & $\mathrm{u}$ & \\
\hline 201.000 & 67.9 & 2.2 & 79 & Caxv & 200.980 \\
\hline 201.123 & 2473.7 & 86.4 & 77 & Fe xIII & 201.121 \\
\hline 201.494 & 187.2 & 7.5 & 65 & $\mathrm{u}$ & \\
\hline 201.557 & 354.3 & 14.6 & 79 & Fe XIII (bl) & \\
\hline 201.743 & 282.4 & 12.6 & 79 & Fe XI (bl Ar XIII?) & 201.734 \\
\hline 201.939 & 84.1 & 3.9 & 79 & $\mathrm{u}$ & \\
\hline 202.047 & 6915.7 & 349.5 & 70 & Fe XIII & 202.044 \\
\hline 202.426 & 356.0 & 20.9 & 79 & Fe xI (bl u) & 202.420 \\
\hline 202.606 & 122.7 & 7.8 & 79 & S VIII (bl Fe xI) & 202.608 \\
\hline 202.711 & 219.5 & 14.4 & 68 & Fe XI (bl Fe XIII) & 202.710 \\
\hline 202.885 & 22.9 & 1.6 & 79 & $\mathrm{u}(? \mathrm{Mn} \mathrm{x})$ & \\
\hline 203.169 & 223.3 & 17.4 & 79 & Fe xIII (bl Fe xII) & 203.173 \\
\hline 203.729 & 359.3 & 33.5 & 79 & Fe XII (bl Fe XIII) & 203.728 \\
\hline 203.825 & 1211.4 & 116.4 & 80 & Fe XIII (sbl) & 203.826 \\
\hline 204.258 & 140.1 & 15.2 & 70 & Fe хIII & 204.263 \\
\hline 204.352 & 26.5 & 3.0 & 79 & u IX-XII & \\
\hline 204.734 & 28.4 & 3.4 & 79 & u VIII (bl oc Fe vIII) & 204.700 \\
\hline 204.939 & 196.8 & 25.2 & 70 & Fe xIII & 204.942 \\
\hline 205.018 & 16.4 & 2.2 & 79 & ? Cr vIII & \\
\hline 205.921 & 29.4 & 4.6 & 77 & Fe XIII & \\
\hline 206.172 & 39.7 & 6.5 & 65 & Fe xI (bl u) & \\
\hline 206.258 & 50.5 & 8.4 & 65 & Fe xI (bl u) & \\
\hline 206.366 & 54.3 & 9.2 & 74 & Fe XII (bl u) & 206.371 \\
\hline 207.449 & 41.6 & 8.5 & 65 & $\mathrm{Fe} \times(\mathrm{bl} \mathrm{u})$ & 207.449 \\
\hline 207.751 & 18.3 & 3.9 & 79 & $\mathrm{Fe} \mathrm{xI}(\mathrm{bl} \mathrm{u})$ & \\
\hline 207.944 & 53.6 & 12.0 & 66 & u XI (bl Ni xI ?) & \\
\hline 208.318 & 22.0 & 5.3 & 65 & Fe xII (bl Ca xv, u) & 208.318 \\
\hline 208.664 & 10.6 & 2.7 & 71 & u (bl Fe хІІІ,Ca xvi?) & \\
\hline 208.694 & 12.8 & 3.3 & 79 & Fe XIII (not Ca xv) & $\mathrm{TN}$ \\
\hline 209.032 & 8.1 & 2.2 & 65 & $\mathrm{u}$ & \\
\hline 209.115 & 25.8 & 7.2 & 65 & Fe xII (bl u) & \\
\hline 209.622 & 54.9 & 16.9 & 65 & Fe xIII & 209.617 \\
\hline 209.771 & 13.2 & 4.1 & 70 & $\mathrm{Fe} x \mathrm{xI}(\mathrm{bl} \mathrm{u})$ & \\
\hline 209.921 & 173.1 & 56.2 & 67 & Fe XIII & 209.919 \\
\hline
\end{tabular}


Table 1. continued.

\begin{tabular}{|c|c|c|c|c|c|}
\hline$\lambda_{\mathrm{o}}$ & DN & Int & $F$ & ID & $\lambda_{\mathrm{r}}$ \\
\hline 211.203 & 16.0 & 6.5 & 79 & $\mathrm{u}$ & \\
\hline 211.321 & 584.6 & 242.3 & 66 & Fe xIV (bl) & 211.316 \\
\hline 211.407 & 22.3 & 9.4 & 79 & u XII (bl Ni xI) & \\
\hline 245.894 & 17.5 & 3.7 & 78 & TN Fe xII & \\
\hline 246.208 & 174.6 & 35.8 & 74 & Fe xIII (bl oc Si vI) & 246.208 \\
\hline 246.372 & 14.9 & 3.0 & 78 & $\mathrm{u}$ & \\
\hline 246.899 & 24.7 & 4.8 & 78 & S XI & 246.895 \\
\hline 247.005 & 11.0 & 2.1 & 78 & TN Fe XII & \\
\hline 247.156 & 27.7 & 5.3 & 78 & S XI (bl Fe XII,u) & 247.159 \\
\hline 247.266 & 17.7 & 3.4 & 78 & $\mathrm{u}$ & \\
\hline 247.404 & 26.0 & 4.9 & 78 & $\mathrm{u}$ & \\
\hline 247.753 & 8.4 & 1.5 & 78 & Fe xIV & 247.760 \\
\hline 248.502 & 30.0 & 5.3 & 78 & $\mathrm{u}(\mathrm{bl} \mathrm{oc} \mathrm{Ov})$ & \\
\hline 249.185 & 73.2 & 12.6 & 77 & Ni xVII (bl Si VI) & 249.177 \\
\hline 249.384 & 81.4 & 13.8 & 78 & TN Fe XII (sbl) & 249.388 \\
\hline 250.678 & 24.4 & 3.7 & 78 & $\mathrm{u}$ & \\
\hline 251.071 & 85.7 & 12.5 & 78 & Fe XVI & 251.074 \\
\hline 251.584 & 13.1 & 1.8 & 78 & $\mathrm{u}$ & 251.580 \\
\hline 251.868 & 40.2 & 5.4 & 77 & Fe XII (bl u) & \\
\hline 251.953 & 491.0 & 65.5 & 77 & Fe XIII & 251.953 \\
\hline 252.198 & 136.5 & 17.8 & 78 & Fe xIV & 252.197 \\
\hline 253.792 & 116.1 & 13.2 & 79 & Si $x$ & 253.795 \\
\hline 253.950 & 13.8 & 1.6 & 79 & $\mathrm{Fe}$ VIII $(\mathrm{bl} \mathrm{Sx})$ & \\
\hline 254.506 & 10.1 & 1.1 & 79 & u (bl oc Fe xvII 254.530) & \\
\hline 254.598 & 71.1 & 7.6 & 67 & Fe XI & 254.6 \\
\hline 254.681 & 18.1 & 1.9 & 79 & $\mathrm{u}$ & \\
\hline 254.841 & 22.8 & 2.4 & 148 & u (bl oc Fe xvII 254.870) & \\
\hline 255.082 & 11.0 & 1.1 & 79 & $\mathrm{~S} x$ (bl u, Fe vIII, oc Fe xxIv) & \\
\hline 255.436 & 15.6 & 1.6 & 79 & $\mathrm{u}$ & \\
\hline 255.755 & 17.9 & 1.8 & 79 & $\mathrm{u}$ & \\
\hline 256.282 & 194.7 & 18.4 & 80 & $\mathrm{He}_{\text {II }}(\mathrm{bl})$ & 256.320 \\
\hline 256.391 & 700.4 & 65.7 & 108 & Si x (bl many) & 256.400 \\
\hline 256.683 & 359.4 & 33.1 & 79 & S XIII & 256.686 \\
\hline 256.926 & 332.1 & 30.1 & 79 & Fe xI (bl Fe xII) & 256.925 \\
\hline 257.151 & 126.3 & 11.2 & 79 & $\mathrm{Sx}$ & 257.150 \\
\hline 257.264 & 614.2 & 54.4 & 69 & Fe $x$ & 257.262 \\
\hline 257.394 & 351.1 & 31.0 & 79 & Fe xIV & 257.300 \\
\hline 257.548 & 205.1 & 17.8 & 79 & Fe XI (sbl) & 257.547 \\
\hline 257.771 & 112.2 & 9.6 & 79 & $\mathrm{Fe} \mathrm{xI}(\mathrm{bl} \mathrm{u})$ & 257.772 \\
\hline 257.913 & 39.6 & 3.4 & 79 & $\mathrm{u}$ & \\
\hline 258.083 & 42.1 & 3.5 & 79 & ? Si Ix (bl u) & 258.082 \\
\hline 258.375 & 804.8 & 66.3 & 79 & Si $\mathrm{x}$ & 258.372 \\
\hline 259.492 & 344.2 & 26.5 & 93 & $\mathrm{~S} x$ (bl Fe xII $)$ & 259.494 \\
\hline 259.747 & 10.7 & 0.8 & 79 & Si ix (bl u, oc Fe xvII) & 259.765 \\
\hline 259.973 & 89.8 & 6.7 & 79 & Fe xII (bl u) & \\
\hline 260.314 & 25.7 & 1.9 & 79 & $\mathrm{u}$ & \\
\hline 261.059 & 539.4 & 37.8 & 84 & Six & 261.056 \\
\hline 261.428 & 15.6 & 1.1 & 79 & $\mathrm{u}$ & \\
\hline 261.741 & 138.8 & 9.4 & 79 & Fe хІІІ & 261.730 \\
\hline 262.702 & 8.2 & 0.5 & 79 & ? Mn xv (bl oc Fe xvII) & 262.7 \\
\hline 262.984 & 358.6 & 22.6 & 78 & Fe xvI (bl Fe xIII) & 262.984 \\
\hline 263.436 & 15.8 & 1.0 & 79 & $\mathrm{u}$ & \\
\hline 263.661 & 16.2 & 1.0 & 79 & $\mathrm{u}$ & \\
\hline 263.803 & 9.8 & 0.6 & 79 & u (bl oc Fe xхIII) & 263.765 \\
\hline 264.234 & 481.0 & 28.5 & 79 & $\mathrm{Sx}(\mathrm{bl} \mathrm{oc} \mathrm{u})$ & 264.233 \\
\hline 264.649 & 22.4 & 1.3 & 79 & $\mathrm{u}$ & \\
\hline 264.787 & 1595.3 & 92.1 & 76 & Fe xIv (bl Fe xI) & 264.787 \\
\hline 265.002 & 47.6 & 2.7 & 79 & Fe xvI & 265.000 \\
\hline 265.746 & 28.7 & 1.6 & 79 & $\mathrm{u}$ & \\
\hline 266.082 & 42.0 & 2.3 & 79 & $\mathrm{u}$ & \\
\hline 266.199 & 10.3 & 0.6 & 79 & $\mathrm{u}$ & \\
\hline 266.370 & 10.3 & 0.6 & 79 & u (bl oc Fe xvII) & 266.410 \\
\hline 266.539 & 13.7 & 0.7 & 79 & $\mathrm{u}$ & \\
\hline 266.602 & 58.3 & 3.1 & 79 & $\mathrm{u}$ & \\
\hline 266.757 & 43.9 & 2.3 & 79 & $\mathrm{u}$ & \\
\hline 267.464 & 14.0 & 0.7 & 79 & ? Ni XIII & 267.468 \\
\hline 267.806 & 29.8 & 1.5 & 119 & ? Ni XI & \\
\hline 269.174 & 11.0 & 0.6 & 79 & $? \mathrm{Mn} \times \mathrm{v}$ & 269.19 \\
\hline 269.489 & 15.4 & 0.8 & 79 & $\mathrm{u}$ & \\
\hline 269.870 & 37.6 & 1.9 & 79 & $\mathrm{u}$ & \\
\hline 270.428 & 39.1 & 2.0 & 79 & ? Mg VI & 270.391 \\
\hline 270.520 & 1063.0 & 53.4 & 72 & $\mathrm{Fe}$ XIV & 270.524 \\
\hline 270.599 & 38.0 & 1.9 & 79 & $\mathrm{u}$ & \\
\hline 270.788 & 20.9 & 1.0 & 79 & Fe xIv (bl u) & 270.80 \\
\hline 271.777 & 24.6 & 1.3 & 79 & $\mathrm{u}$ & \\
\hline 271.990 & 689.7 & 35.3 & 86 & Si x (bl u) & 271.992 \\
\hline 272.105 & 15.9 & 0.8 & 77 & $\mathrm{u}$ & \\
\hline 272.182 & 37.6 & 1.9 & 74 & Fe XIII & \\
\hline 272.538 & 28.2 & 1.5 & 79 & u (bl Fe xII) & \\
\hline 272.657 & 37.9 & 2.0 & 79 & Si VII & 272.660 \\
\hline 272.884 & 17.1 & 0.9 & 79 & $\mathrm{u}$ & \\
\hline 273.031 & 14.7 & 0.8 & 79 & u (bl Fe xIv) & \\
\hline
\end{tabular}

Table 1. continued.

\begin{tabular}{|c|c|c|c|c|c|}
\hline$\lambda_{\mathrm{o}}$ & DN & Int & $F$ & ID & $\lambda_{\mathrm{r}}$ \\
\hline 273.169 & 18.5 & 1.0 & 79 & $\mathrm{u}$ & \\
\hline 273.552 & 20.0 & 1.1 & 79 & Fe XIV & $\mathrm{N}$ \\
\hline 274.203 & 2516.2 & 139.9 & 81 & Fe xIV (bl Si viI) & 274.203 \\
\hline 275.364 & 93.7 & 5.6 & 79 & $\mathrm{Si}$ VII & 275.370 \\
\hline 275.694 & 19.5 & 1.2 & 79 & Si vII (bl u) & 275.690 \\
\hline 276.143 & 15.4 & 1.0 & 79 & Mg VII & 276.145 \\
\hline 276.459 & 9.9 & 0.6 & 79 & $\mathrm{u}$ & \\
\hline 276.852 & 62.1 & 4.1 & 79 & Si vIII bl Si VII & 276.839 \\
\hline 277.040 & 154.5 & 10.4 & 117 & Si VIII (bl Mg VII) & 277.000 \\
\hline 277.260 & 393.9 & 27.1 & 87 & $\operatorname{Six}$ & 277.265 \\
\hline 278.154 & 11.1 & 0.8 & 79 & Si x (bl u) & \\
\hline 278.400 & 80.1 & 6.1 & 118 & Mg VII (bl Si VII) & 278.395 \\
\hline 278.706 & 11.5 & 0.9 & 79 & ? Ni XI & \\
\hline 279.479 & 12.3 & 1.1 & 79 & $\mathrm{u}$ & \\
\hline 280.144 & 17.7 & 1.6 & 125 & Al Ix (bl u oc Fe xviI) & 280.160 \\
\hline 281.411 & 109.1 & 11.5 & 77 & $\mathrm{~S}_{\mathrm{XI}}$ & 281.401 \\
\hline 282.418 & 20.5 & 2.4 & 77 & $\mathrm{Al}_{\text {IX }}$ & 282.422 \\
\hline 282.990 & 14.3 & 1.8 & 77 & $\mathrm{u}$ & \\
\hline 283.468 & 26.7 & 3.6 & 77 & Fe xII (bl u) & \\
\hline 283.779 & 11.2 & 1.5 & 77 & $\mathrm{u}$ & \\
\hline 283.943 & 21.7 & 3.0 & 77 & u (bl Fe xvII) & 283.930 \\
\hline 284.044 & 76.6 & 10.9 & 72 & $\mathrm{Al}$ Ix (bl u oc Fe XVII) & 284.042 \\
\hline 284.159 & 3999.0 & 576.6 & 81 & $\mathrm{Fe} x v(\mathrm{bl})$ & 284.160 \\
\hline 284.618 & 13.7 & 2.1 & 77 & $\mathrm{u}$ & \\
\hline 285.594 & 31.2 & 5.3 & 77 & $\mathrm{~S} \mathrm{XI}_{\mathrm{XI}}$ & 285.587 \\
\hline 285.832 & 47.3 & 8.2 & 94 & $\mathrm{~S} \times \mathrm{XI}$ & 285.822 \\
\hline 286.376 & 9.8 & 1.8 & 77 & Al IX & 286.380 \\
\hline 287.872 & 6.7 & 1.5 & 77 & Fe xIV & 287.870 \\
\hline 288.158 & 11.9 & 2.7 & 77 & NixvI & 288.160 \\
\hline 288.407 & 58.7 & 13.7 & 84 & $\mathrm{~S}$ XII & 288.400 \\
\hline 288.548 & 9.9 & 2.3 & 76 & Fe xIII & 288.54 \\
\hline 289.152 & 26.3 & 6.7 & 77 & Fe xIV & 289.148 \\
\hline 290.703 & 22.0 & 6.8 & 90 & Si Ix (bl oc Fe viI) & 290.687 \\
\hline 291.010 & 23.6 & 7.5 & 65 & Fe XII & 291.010 \\
\hline
\end{tabular}

Notes. $\lambda_{0}(\AA)$ is the measured wavelength. DN is the number of total counts in the line, while Int is the calibrated intensity (phot $\mathrm{cm}^{-2} \mathrm{~s}^{-1} \operatorname{arsec}^{-2}$ ). $F$ is the full-width-half-maximum in $\mathrm{m} \AA$ (wide lines are clearly blended) while the column ID provides the identification (bl: blended; sbl: self-blend; u: unidentified; bl oc: blended in other plasma conditions). In a few cases, the class of a line is given (i.e.: u VII is a line with a morphology similar to Fe VII), as well as the rest wavelength $\left(\lambda_{\mathrm{r}}, \AA\right)$ from the literature. "N" indicates a new identification proposed here, while "T N" a tentative new one.

hence the contribution of cooler TR lines is more reduced. The averaged EIS spectrum is shown in Fig. A.1. Table A.1 provides the list of the strongest lines present in this spectrum.

Figure 5 shows the EM loci curves, which indicate a nearly isothermal plasma around $\log T[\mathrm{~K}]=6.15$. Strong decays to the ground states were selected for iron (Fe viII 185.2, Fe x 184.5, Fe xII 192.4, Fe XIII 202.0, Fe xIV 264.8, Fe xv 284.2, Fexvi $263 \AA)$ sulfur ( $\mathrm{Sx}$ 264.2, S xI 281.4, S XII 288.4, S XIII $256.7 \AA$ ) and argon (Ar XI $188.8 \AA$ ). The curves are displayed with the Asplund et al. (2009) photospheric abundances, with the exception of argon, increased by a factor of two.

A few exposures were averaged to obtain a series of good spectra along the slit, crossing the solar limb. Figure A.2 shows the intensities in a few selected lines as they vary across the solar limb, to illustrate the approximate formation temperature of some of the unidentified lines. This is a rough estimate, considering that even lines from the same ion do have different profiles because of the varying density and temperature across the limb.

\section{Discussion}

A summary of the main findings based on the analysis of both off-limb observations now follows. The atomic data are either mentioned or are from CHIANTI v.6 (Dere et al. 2009). The in- 

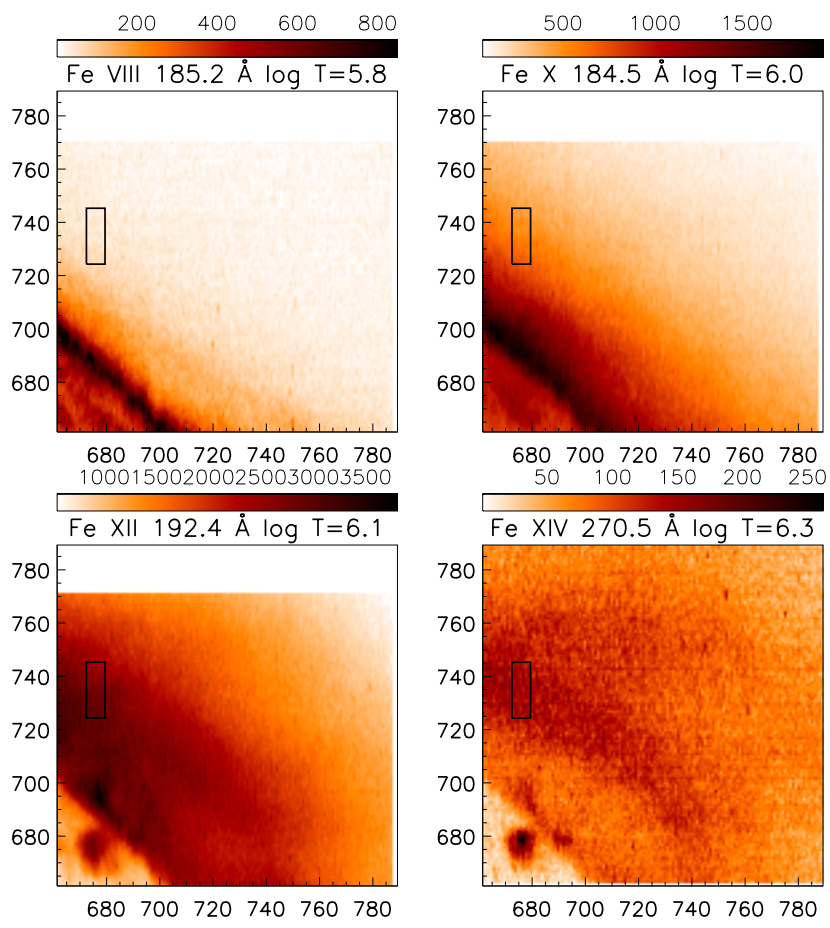

Fig. 4. Monochromatic images (negative, integrated counts) of some of the strongest lines observed by EIS on 2007 March 11 and formed over a range of temperatures. The box represents the area chosen to obtain a sample spectrum, shown in Fig. A.1. The coordinates are solar $X$ and solar $Y$, in arcseconds from disk centre.

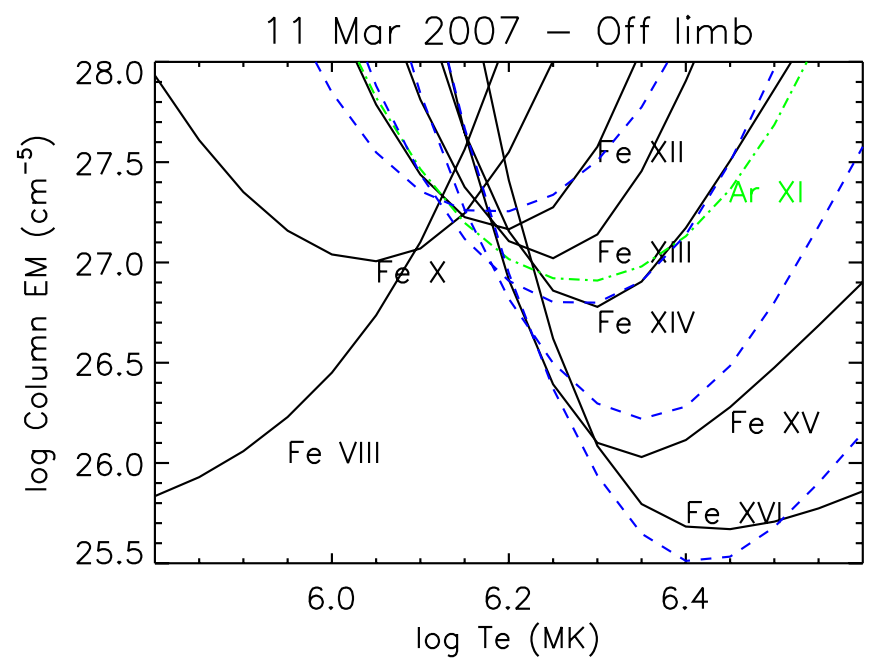

Fig. 5. EM loci curves of the 2007 March 11 off-limb region for Ar XI (dot dash), and a selection of iron (solid lines), and sulfur (dashed) ions, using the Asplund et al. (2009) photospheric abundances, with the exception of argon, increased by a factor of two.

dices and spectroscopic notation for each transition can be found in the cited papers or directly within the CHIANTI database.

\subsection{Cooler lines}

The atomic data and identifications for Fe viI have been presented in Witthoeft \& Badnell (2008) and Del Zanna (2009a), although some contradicting line identifications were presented at the same time by Young \& Landi (2009). Given this uncertainty, the Witthoeft \& Badnell (2008) atomic data are not included in CHIANTI v.7. The atomic data and identifications for Fe vIII

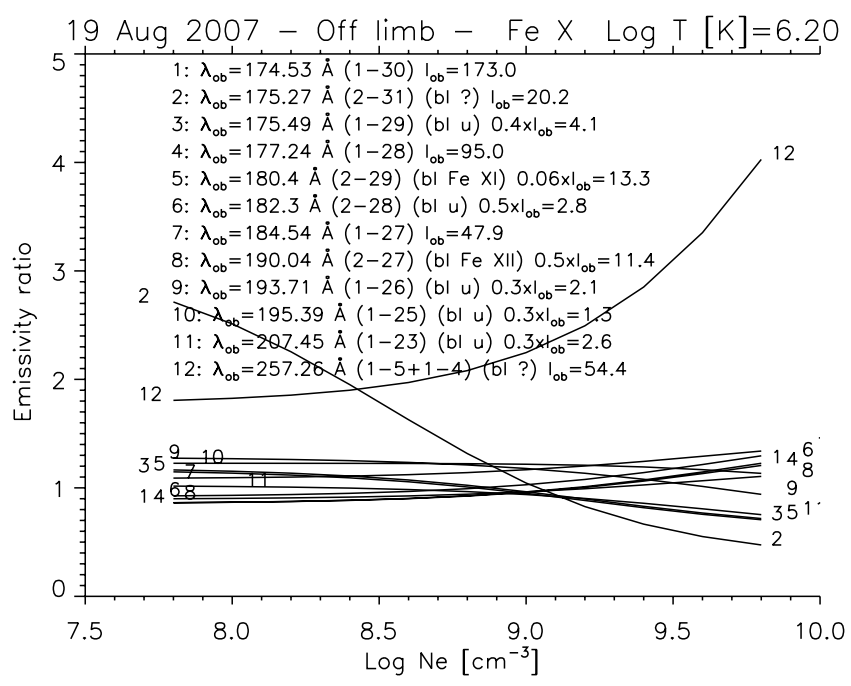

Fig. 6. Emissivity ratio curves relative to the main Fe $x$ EUV transitions observed by Hinode EIS on 2007 Aug. 19.

have been discussed in Del Zanna (2009b) and have been included in CHIANTI v.7. There is hardly any Fe vII residual emission in the off-limb spectra and the Fe vIII lines are weak.

The CHIANTI atomic data for Fe IX are from Storey et al. (2002). Young (2009) identified four new lines. The three main decays from the $3 \mathrm{~s}^{2} 3 \mathrm{p}^{4} 3 \mathrm{~d}^{2} .{ }^{3} G_{4,5,3}$ were confirmed by Del Zanna (2009a). The fourth line was identified by Young (2009) with the main decay from the $3 s^{2} 3 p^{5} 4 p$ configuration (13-140), observed at $197.862 \AA$ A. Del Zanna (2009a) provided a different tentative identification for the 13-140 transition, as the $194.784 \AA$ line. This identification appears to be incorrect, given that in the off-limb spectra the observed line is too weak. There are no other observed levels from the $3 s^{2} 3 p^{5} 4 p$ configuration, so it is not easy to identify any lines originating from this configuration.

There are consistent discrepancies between the strongest 171.0 $\AA$ resonance line and the other strong Fe IX lines in the sense that the 171.0 $\AA$ has an observed intensity too high compared to the predicted one. This could be caused by a problem in the EIS calibration at the very edge of the EIS responsivity in the SW channel. More analysis is in progress.

Finally, the cooler emission that is residually present in the spectra and caused by Si vi, Si viI, Si vIII, Mg v, Mg vI, Mg vII, O vI, and Ov is discussed in Del Zanna (2009a).

\section{2. $\mathrm{Fex}$}

The electron scattering calculations adopted within CHIANTI for this ion are from Del Zanna et al. (2004). The identifications are also from the benchmark work described in that paper, where most lines were identified. Many weaker transitions were only tentatively identified, however, given the paucity of high-resolution solar spectra available at the time. All identifications of lines in the Hinode/EIS wavelengths proposed by Del Zanna et al. (2004) are now confirmed.

The emissivity ratio curves relative to the Fe x EUV transitions observed by Hinode EIS are shown in Fig. 6. The curves indicate very good agreement between theory and experiment, with the exception of the $257.26 \AA$ self-blend (identified in Del Zanna et al. 2004), which has a temperature sensitivity and also a slight density dependence above $\log N_{\mathrm{e}}\left[\mathrm{cm}^{-3}\right]=9$. 

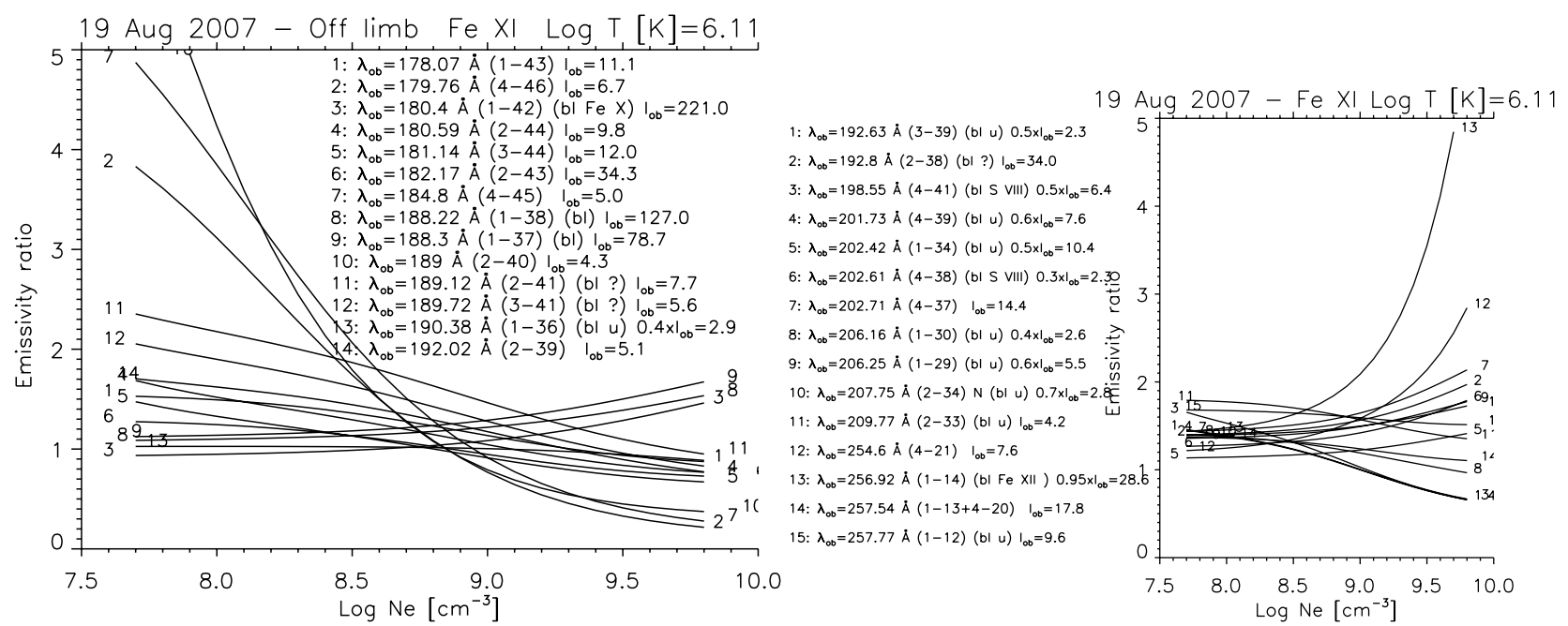

Fig. 7. Emissivity ratio curves relative to the main Fe xi EUV transitions observed by Hinode EIS on 2007 Aug. 19.
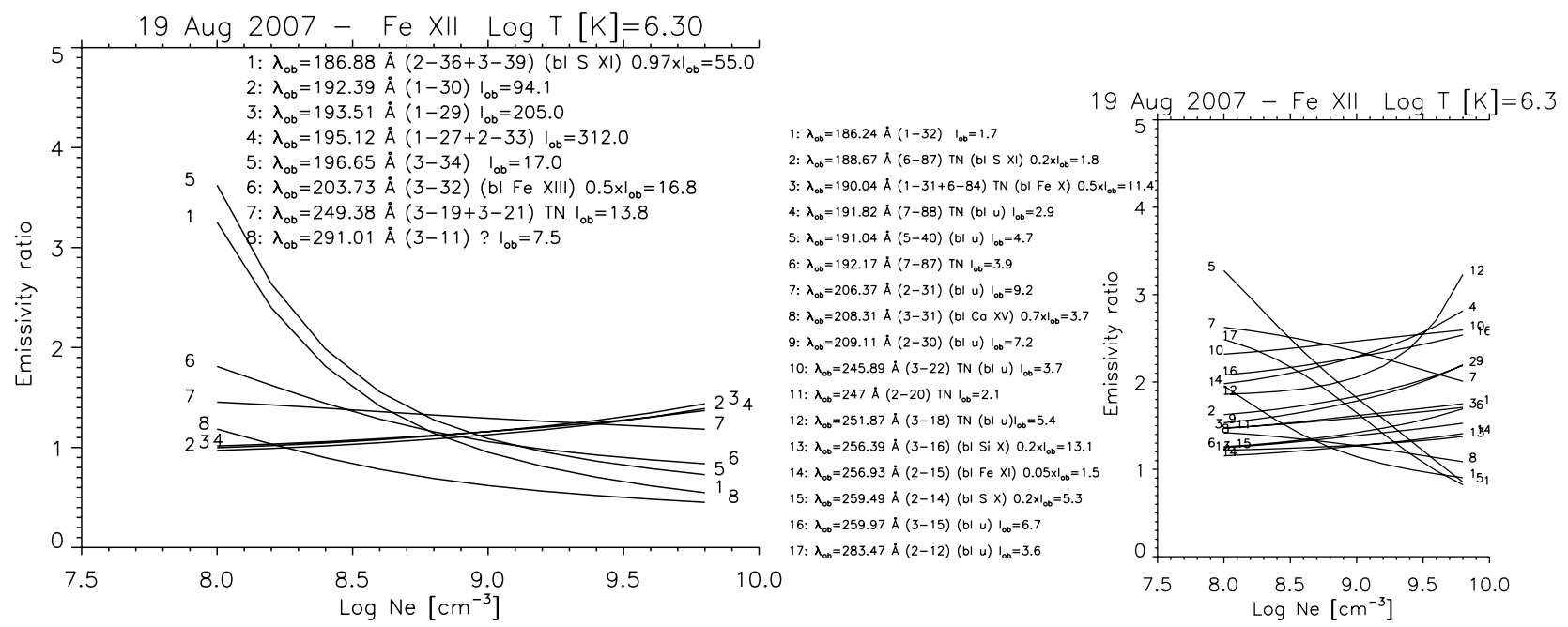

Fig. 8. Emissivity ratio curves relative to the main Fe xII EUV transitions observed by Hinode EIS on 2007 Aug. 19.

\section{3. $\mathrm{FexI}$}

This ion has been the most complex of all coronal ions. Final agreement between observation and theory has been achieved after a few years of work along the S-like sequence. The R-matrix scattering calculation performed as part of the Iron Project is described in Del Zanna et al. (2010). The new calculation, obtained with an optimal target, finally allowed the identifications of most transitions. The details are given in Del Zanna (2010), and the data have been made available within CHIANTI v.7. Fe XI lines represent a good fraction of all the coronal lines in the Hinode/EIS spectra, so solving the problems for this ion was necessary for this work. Figure 7 gives one example of emissivity ratio curves for this ion, confirming the results presented in Del Zanna (2010). There are some interesting temperature diagnostics for this ion, discussed in Del Zanna (2010). There is an ample range of line ratios that can be used to measure the electron density, as Fig. 7 shows.

\subsection{FexII}

CHIANTI adopted for Fe XII ( $n=3$ configurations) the Storey et al. (2005) R-matrix electron collisional data and the new identifications of Del Zanna \& Mason (2005). For the first time, good agreement between theory and observations for the strongest lines was found, and most lines were identified. As in the Fe x case, many weaker transitions were only tentatively identified.

Almost all of the proposed identifications for the Hinode/EIS wavelengths are now confirmed (see Fig. 8). A few new tentative identifications are proposed here. Del Zanna \& Mason (2005) gave a tentative suggestion for the identification of the 6-84 line, as a self-blend with the 5-40, observed at $191.05 \AA$. The $6-84$ is the strongest decay from the $3 \mathrm{~s} 3 \mathrm{p}^{3} 3 \mathrm{~d}$ configuration, from the ${ }^{4} \mathrm{D}_{7 / 2}$. In the present spectra the $191.05 \AA$ line is well observed, and it is true that the predicted intensity of the 5-40 transition is not sufficient to explain the observed intensity, but this line cannot be a self-blend with the 6-84 line because it is too weak. The energy difference between observed and theoretical values for the other configurations suggests a wavelength around $188 \AA$. There are a few possibilities, in particular that the line is blended with the strong Fe XI 188.2, 188.3 $\AA$ lines, but a few other decays from the ${ }^{4} \mathrm{D}$ should be observable with the present deep exposures.

A search over many possibilities has resulted in one reasonable option, namely that the $6-84$ is blended with the Fex $190.04 \AA$ line. The residual intensity is about right. The difference between observed and theoretical energies is about $17500 \mathrm{~cm}^{-1}$. The decay from the ${ }^{4} \mathrm{D}_{5 / 2}$ level (87) to level 7 is the 

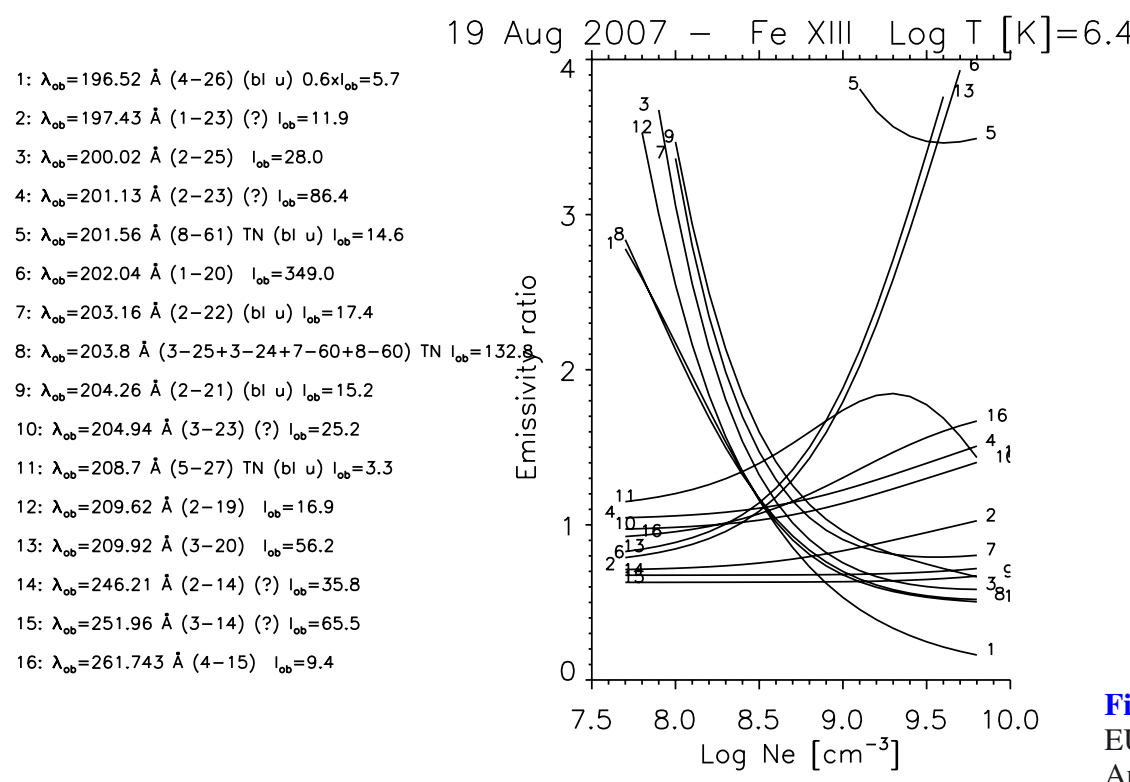

Fig. 9. Emissivity ratio curves relative to the main Fe XIII EUV transitions observed by Hinode EIS on 2007 Aug. 19. second strongest line from this configuration, and there is a line at the predicted wavelength $\left(192.17 \AA\right.$, from the ${ }^{4} \mathrm{D}$ theoretical splittings) and intensity, although it would be blended with a cooler line on-disk. The 6-87 (weaker) line would be blended at $188.68 \AA$. The weak decays from the ${ }^{4} \mathrm{D}_{3 / 2}$ (level 88 ) to levels 8,7 should be observable, and would be at 193.41 and $191.82 \AA$ respectively. The former would be blended, while the latter has the proper wavelength and about the correct intensity.

The best density diagnostic is the ratio of the two self-blends at 186.88 and $195.12 \AA$, both of which were identified as such for the first time by Del Zanna \& Mason (2005). At high densities, the $196.65 \AA$ is the best choice, although this line is blended in on-disk observations.

\subsection{FexIII}

The most accurate collisional data for this ion have recently been published by Storey \& Zeippen (2010), as part of the Iron Project. These data have been benchmarked by Del Zanna (2011), where a number of new identifications have been suggested. These data have been made available within CHIANTI v 7. Figure 9 shows the emissivity ratio curves relative to the main Fe XIII EUV transitions observed by Hinode EIS on 2007 Aug. 19. The Fe XIII lines discussed here are not significantly temperature-dependent, i.e. similar emissivity ratios are obtained for different temperatures. The observed intensities of the 246.2 and $251.95 \AA$ are too low by a factor of 1.5 because of an EIS calibration problem (see Del Zanna 2011 and below).

Del Zanna (2011) suggested two possible identifications for a new strong self-blend (7-60, 8-60): either this self-blend is blending the FexI $202.42 \AA$ or the important self-blend at $203.82 \AA$. Evidence that supports both choices has been found, with the quiet-Sun off-limb measurements favouring the first option, and the active region ones favoring the latter, which is adopted here. In either case, the 4-26 196.52 A line appears to be blended in both off-limb spectra. The latter choice lets the emissivity ratio of the $203.82 \AA$ blend agree very well with those of the 202.04, 209.62, 209.92, $261.74 \AA$ lines, providing densities of $\log N_{\mathrm{e}}=8.5$ and 8.35 for the active region and quiet Sun, both measurements being very close to those obtained from $\mathrm{Six}$, but slightly lower than Fe XII. The slightly higher emissivity ratio curves for the Fe XIII 200.02, 204.26, 203.16 $\AA$ lines could mean that these (weaker) lines are blended. Laboratory measurements for this ion are necessary to clarify the various blends.

\subsection{FexIV}

Significant discrepancies between observed and predicted intensities of the very strong Fe XIV EUV coronal lines existed until the calculation of Storey et al. (2000). Liang et al. (2010), as part of the UK APAP network, have performed a much more extensive R-matrix calculation that has been made available within CHIANTI v.7 and is adopted here.

Figure 10 shows the emissivity ratio curves for the off-limb active region spectrum, where the Fe xIV lines are very strong. There are obvious discrepancies between observed and predicted intensities, in particular when branching ratios are considered, as also noticed in Liang et al. (2010). The $211.3 \AA$ is at the edge of the SW channel, and therefore it makes sense that the radiometric calibration at the edge is not correct. Notice that the very nearby Fe XIII lines do agree well, so the problem seems to be just at the edge. There appears to be another calibration problem for lines around $250 \AA$, as also found looking at the Fe xIII lines. The comparison improves if the intensities of the lines around $250 \AA$ is increased by $20 \%$.

Malinovsky \& Heroux (1973) published an excellent rocket EUV spectrum, radiometrically calibrated with an accuracy of 10-20\% for lines close in wavelength. Figure 11 shows the emissivity ratio curves from their data, with the Si vII contribution removed from the $274.20 \AA$ line. The agreement is impressive, within a relative $10 \%$ for all strong lines, and confirms the Hinode/EIS calibration problem around $250 \AA$.

Several very weak transitions are tentatively identified as Fe xIV. The benchmark work on Fexi (Del Zanna 2010) has suggested the presence of a new Fe XI line blending the strong Fe XIV 264.79 A line. Indeed, in the quiet Sun off-limb spectrum the Fe xIV lines are very weak, but the Fe XI are not, and excellent agreement between observed and predicted intensities for both Fe XIV and Fe XI is obtained considering that Fe XI contributes a significant $20 \%$ to the blend. The width of the $264.79 \AA$ line is indeed broader than the widths of the other Fe xiv lines. The exact wavelength of the Fe XI line is difficult to assess. Laboratory 
G. Del Zanna: Benchmarking atomic data for the CHIANTI atomic database: coronal lines observed by Hinode EIS

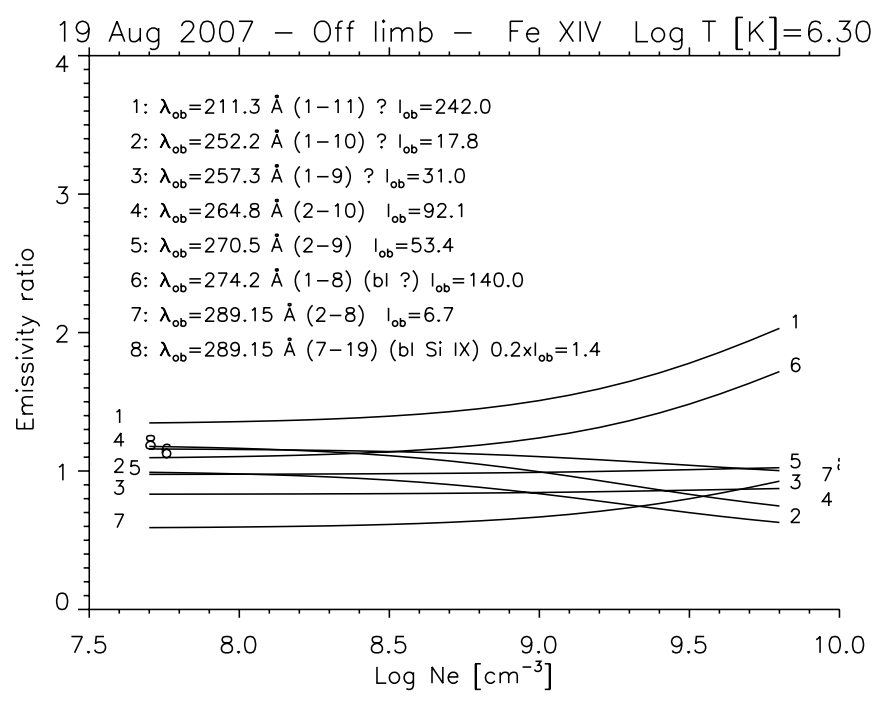

Fig. 10. Emissivity ratio curves relative to the Fe xIv EUV transitions observed by Hinode EIS on 2007 Aug. 19.

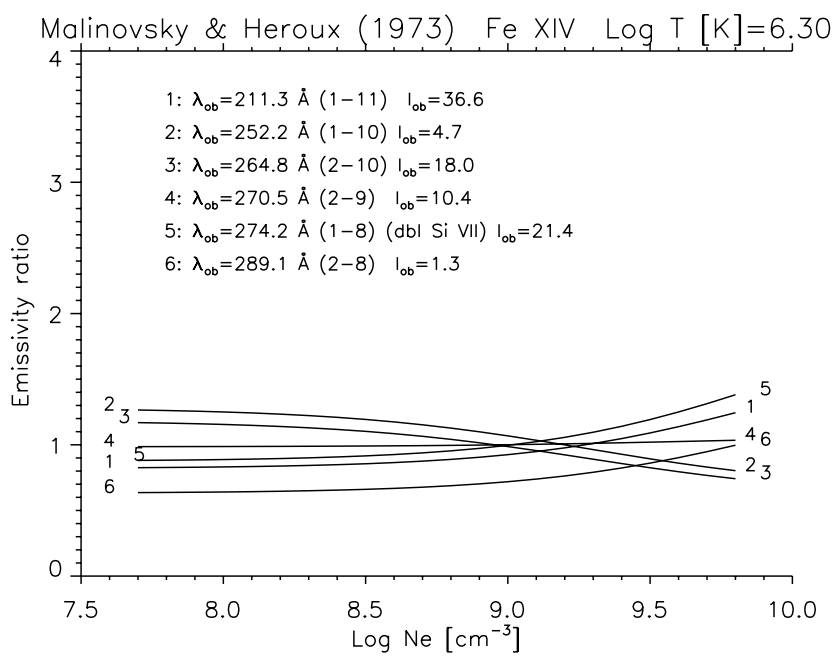

Fig. 11. Emissivity ratio curves relative to the Fe xIv EUV transitions observed by Malinovsky \& Heroux (1973).

measurements are needed. The presence of the Fe xI line could affect Doppler measurements of the $264.79 \AA$ line. The best density diagnostic for this ion is the ratio of the 264.79 and $274.20 \AA$ lines, although both lines are blended.

\subsection{Fexvı and FexvII}

For Fe xvi the atomic data are from Sampson et al. (1990). There are only three lines at the EIS wavelengths, the strongest of which is the $262.98 \AA$ line. Observed and predicted intensities agree well, but again the branching ratio between the 251.06 and the $265 \AA$ lines indicates that the $251.06 \AA$ is too weak by $20 \%$, again confirming the Hinode/EIS calibration problem.

It is interesting to notice that the EM loci plots (see Fig. 3) indicate that the Fe XVI emission measure is much lower than what it should be, unless the $\mathrm{S} / \mathrm{Fe}$ abundance changes with temperature. This is difficult to establish, given that Fe xvI is one of the "anomalous ions" discussed in a series of papers (see the review in Del Zanna et al. 2002). See the discussion on the calcium lines below.

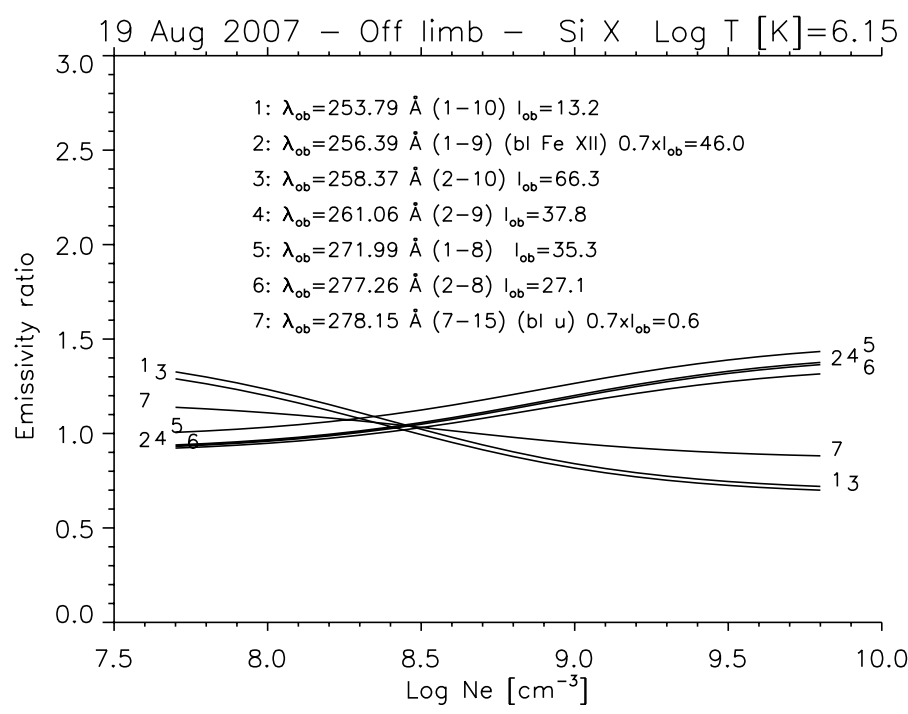

Fig. 12. Emissivity ratio curves relative to the Six EUV transitions observed by Hinode EIS on 2007 Aug. 19.

For Fe XVII the scattering data calculated as part of the UK APAP network by Liang \& Badnell (2010), together with the identifications of the EUV lines described in Del Zanna \& Ishikawa (2009), have been made available within the CHIANTI database v.7 and were used for the present study.

There is no clear evidence of any measurable Fe XVII emission in the active region spectrum. This is a significant result because it puts constraints on active-region heating models. Various weak coronal lines are present at the same wavelengths as the Fe XVII lines, as already described in Del Zanna \& Ishikawa (2009).

\subsection{Ca ions}

The Ca XIV lines are difficult to assess because two out of the three strongest lines are significantly blended. The $183.46 \AA$ line is a known blend with at least ArxIv. The 186.61 $\AA$ line is a known blend with at least a strong Fe vIII transition. The only line possibly not blended is the strongest, at $193.87 \AA$. However, the estimate of the Fe VIII contribution from the $186.61 \AA$ line suggests that the $193.87 \AA$ is blended as well.

Several Caxv transitions should be observable with Hinode/EIS, at 200.97, 208.72, 181.90, 176.93, 208.32. However, it appears that all lines, with perhaps the exception of the strongest one at $200.97 \AA$, contribute very little (less than $20 \%$ ) to the observed intensities.

\subsection{Si ions}

There are a few weak Si Ix transitions in the EIS wavelengths. The strongest, predicted at $290.69 \AA$ and observed at $290.70 \AA$ is possibly the only un-blended line from this ion. The others, at $258.08,259.76,261.43 \AA$ are all blended according to the present atomic data.

Liang et al. (2009), as part of the UK APAP network, has produced an extensive R-matrix calculation for $\mathrm{Six}$, finding significant differences with previous calculations. This ion is potentially very important for Hinode/EIS because it provides some of the best density diagnostics, which turn out to be slightly modified when the new atomic data, now made available through CHIANTI v.7, are used. The new atomic data for Si x provide 


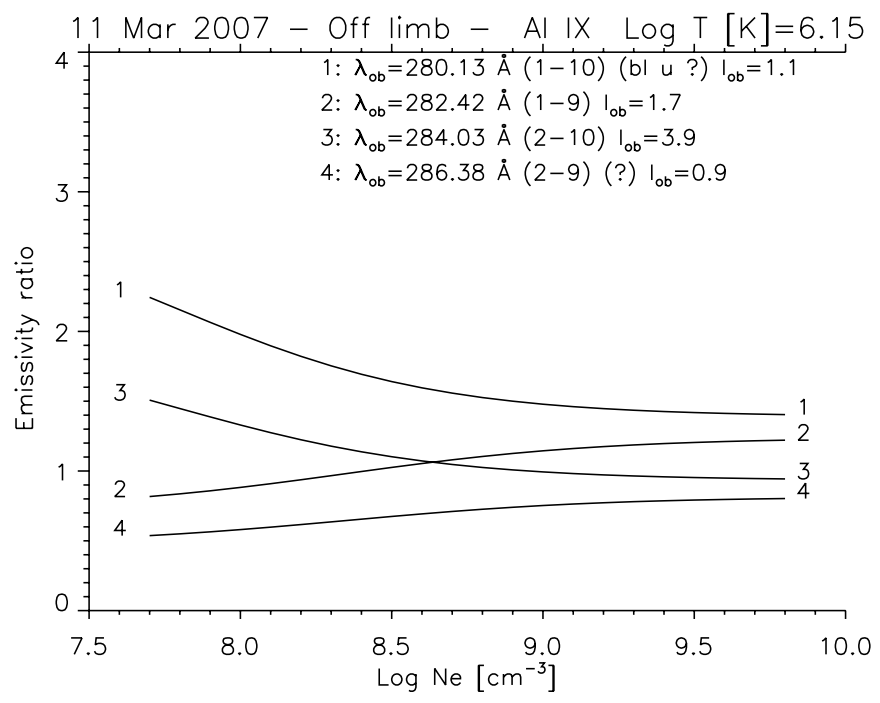

Fig. 13. Emissivity ratio curves relative to the Al Ix EUV transitions observed by Hinode EIS on 2007 Mar. 11.

an impressive agreement between predicted and observed intensities, as Fig. 12 shows. The best density diagnostics are the 258.37 and 261.06 lines, because they are strong, unblended and nearby.

\subsection{Al Ix}

The strongest of the $\mathrm{Al}$ Ix lines at $284.02 \AA$ is particularly important because it is in the blue-wing of the Fe xv 284.16 $\AA$ resonance line. The $\mathrm{Al} \mathrm{Ix}$ is a strong line, and work on the Fe xv line profile should take it into account. In active region conditions, the $\mathrm{Al}$ Ix line is blended in the blue-wing of the Fe Xv and is difficult to measure. The quiet Sun off-limb spectrum is better suited to study the Al Ix lines. The emissivity ratio curves for that observation are shown in Fig. 13. Excellent agreement for the 282.42 and $284.02 \AA$ is found, providing a density in line with what was found from the other ions. The very weak 280.14, which would blend an Fe xvII self-blend first identified in Del Zanna \& Ishikawa (2009), appears to be even more blended in these quiet Sun conditions. The strong Al Ix $284.02 \AA$ line is also blended in solar flares with an Fe XvII line identified for the first time in Del Zanna \& Ishikawa (2009).

\subsection{1. $\operatorname{Mg} x$}

Brown et al. (2008) suggested the presence of a few $\mathrm{Mg}$ x lines in the EIS spectra. In the off-limb observations analysed here, there is no clear evidence of any significant $\mathrm{Mg}$ x emission. If the very weak $190.56 \AA$ line is entirely caused by $\mathrm{Mgx}$, then the other 187.07 and $187.18 \AA$ lines are significantly blended.

\subsection{Ni ions}

It is probable that a few $\mathrm{Nix}$ are present in the EIS spectra. Regarding Ni xI, Brown et al. (2008) lists a few possible Ni xI lines in the EIS spectra, and Landi \& Young (2009) identified a few transitions, despite noting some unexplained inconsistencies, which could be caused by problems with the atomic data for this ion. The present observations indicate that most Ni XI lines are blended, but the benchmark is complicated by the presence of some unidentified transitions. At first sight, the strongest Ni XI is the 1-4 transition, with a NIST wavelength of

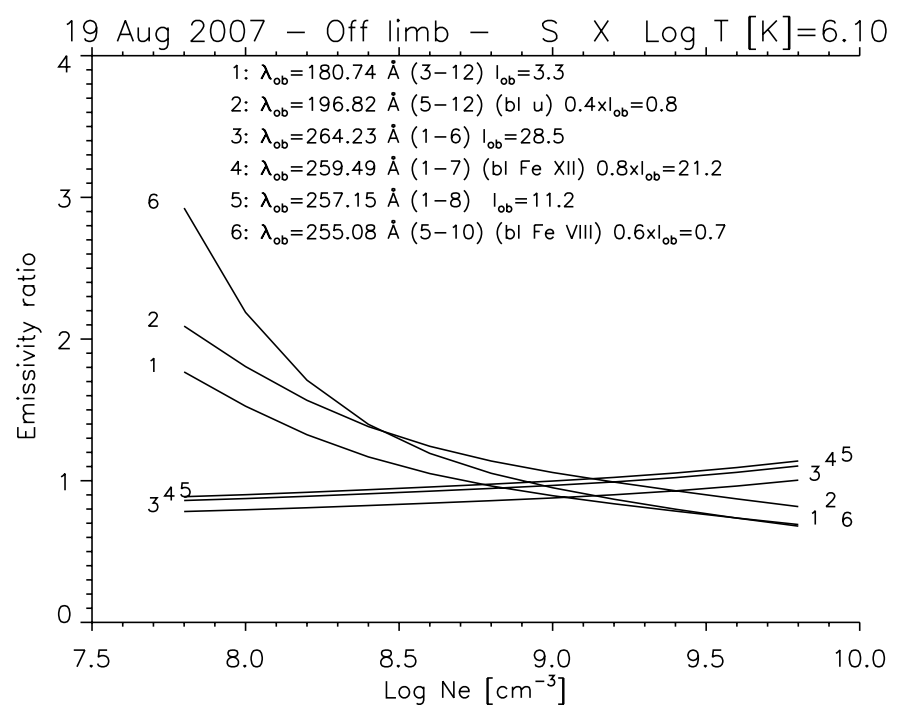

Fig. 14. Emissivity ratio curves relative to the S x EUV transitions observed by Hinode EIS on 2007 Aug. 19.

$207.93 \AA$. There is indeed an observed line in the EIS spectra at $207.94 \AA$. Assuming that this line is caused entirely by Ni XI would predict that other Ni xi transitions (1-10: $186.98 \AA$; $1-7$ : $198.38 \AA$; 1-3: $211.43 \AA$ ) are blending some observed lines. However, there are two still unidentified lines (4-16 and 5-16) that should be well observable in the EIS LW channel, around 270 and $280 \AA$. There are no observed lines with the required strength, so the only option appears to be that they would blend the Six $271.99 \AA$ and the S xI $281.41 \AA$. The Six is indeed a wide line and could be blended, but the current atomic data for S XI do not indicate that the $281.41 \AA$ is blended. Therefore, with the current atomic data for Ni XI and S XI, the only explanation is that all the NixI are blended. A more in-depth benchmark of $\mathrm{Ni} \mathrm{x}$ and $\mathrm{Ni} \mathrm{XI}$ lines is left to a future paper.

In the case of Ni XII, Ni XIII and Ni XIV it appears that most lines, if present, are blended. For Ni xv the only line that might be observed is the strongest one at $176.74 \AA$. For Ni XVI the only line which might not be blended is the very weak $198.31 \AA$ transition. Three other lines (194.02, 195.27, 185.23 $\AA$ ) provide significant contributions to blended lines, the main one being the $185.23 \AA$ one $(80 \%$ of the observed line caused by Ni XVI, the rest by Fe vIII). The $195.27 \AA$ forms a branching ratio with the $185.23 \AA$ one, and could be used in principle to remove the $\mathrm{Ni}$ XVI contamination from the Fe VIII in on-disk active region observations, but the presence of a further unidentified coronal line complicates the matter.

\subsection{S ions}

The comparison between predicted and observed intensities for $\mathrm{Sx}$ is satisfactory once all the blends are taken into account, as Fig. 14 shows. The strongest and unblended line is the 1-6 264.23 $\AA$ line. The 3-12 180.73 $\AA$ line can be used to measure the electron density. The values obtained agree well with those from ions formed at similar temperatures.

The strongest and unblended line for S xI is the 1-8 $281.40 \AA$ line. It can be used to obtain the electron density in combination with the $2-7285.82 \AA$ or with the $3-12246.89 \AA$ line, as Fig. 15 shows. The densities obtained are close to those obtained from ions formed at similar temperatures. Many lines for this 


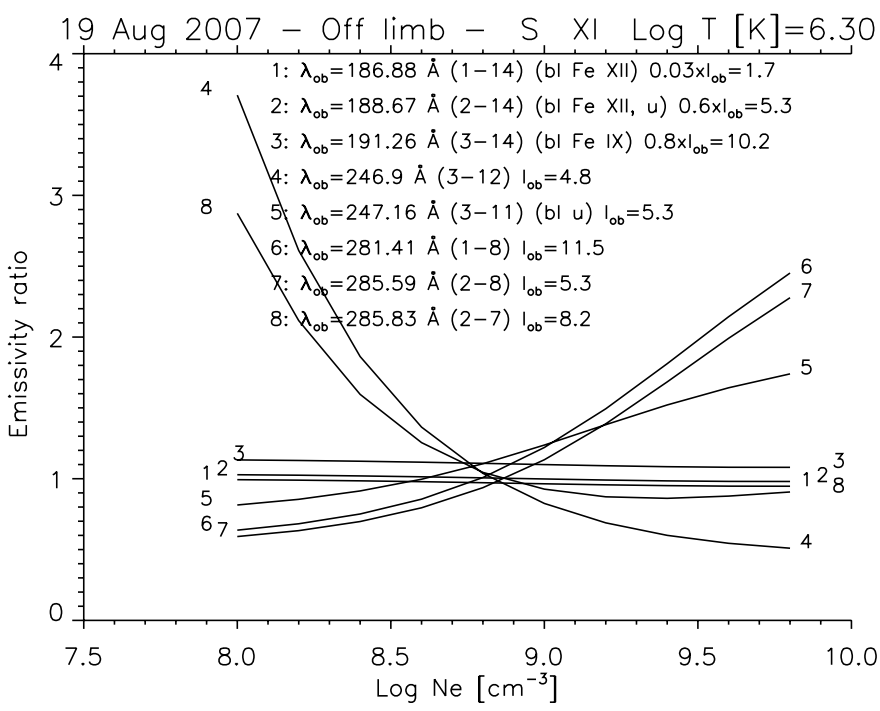

Fig. 15. Emissivity ratio curves relative to the S xi EUV transitions observed by Hinode EIS on 2007 Aug. 19.

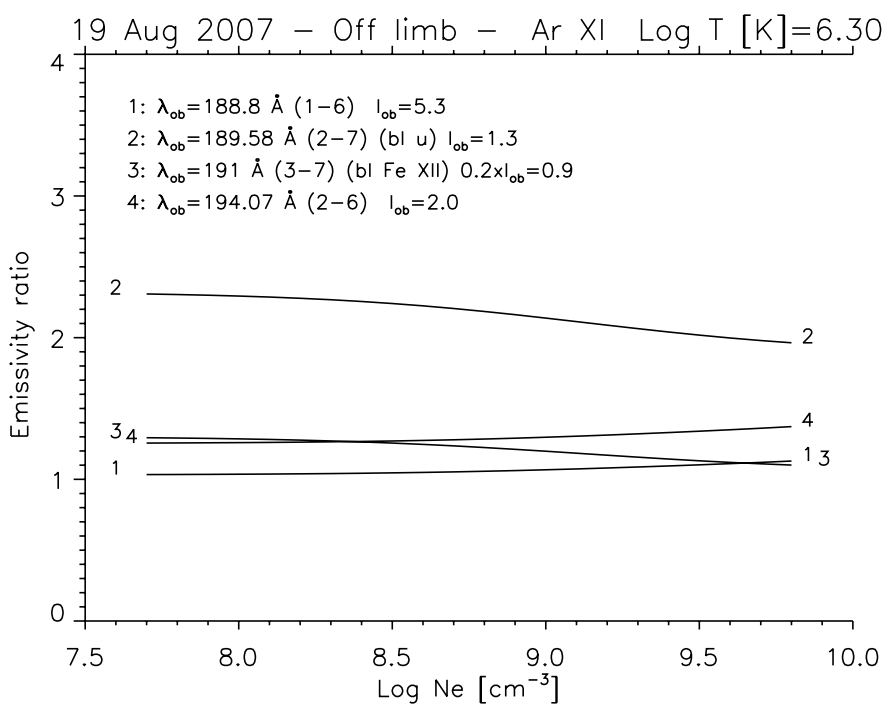

Fig. 16. Emissivity ratio curves relative to the Arxi EUV transitions observed by Hinode EIS on 2007 Aug. 19.

ion are blended. The most notable one is the $1-14$ because it blends the most important density diagnostic for Hinode/EIS, the Fe XII $186.88 \AA$. There have been reports that densities obtained from this line are too high, which could be explained by the presence of blends. For both off-limb cases, the S XI contribution to the Fe XII $186.88 \AA$ line is very low ( $3 \%$ or less), so any additional significant blending could only come from some lines that become bright at high-densities.

There is only one line from S xII, observed at $288.41 \AA$, so it is difficult to assess if any blending is present. The same is true for the strong S XIII $256.7 \AA$ line, although the emission measures for both these lines (cf. Fig. 3) suggest that there is no blending.

\subsection{Arions}

Brown et al. (2008) suggested the presence of a few weak Ar xI lines in the EIS spectra. Figure 16 shows that in the off-limb active region spectra the only lines that are possibly not blended are the strongest one, at $188.806 \AA$, and the weaker $194.1 \AA$ line (within 20\%). These lines are intrinsically weak, but are well observed by Hinode/EIS, given the fact that they are located not far from the peak sensitivity of the multi-layer coatings for the SW channel.

Ar XIV has a typical formation temperature of $\log T[\mathrm{~K}]=$ 6.55 , and a few lines are observable with EIS in active region spectra. Unfortunately, the analysis has shown that all lines seem to be significantly blended, with the exception of the 194.40 A line. These Ar XI and Ar xIV lines are potentially very important for studying the relative argon abundance in active regions.

\section{Relative abundances}

The EM loci curves offer the possibility to estimate the abundances of a few elements relative to iron. The active region EM loci curves (Fig. 3) suggest that either the strongest Ca XIV and $\mathrm{Caxv}$ lines are blended or that the calcium abundance is too low. Alternatively, the strongest lines are not blended, the calcium abundance is correct, the emission measure for Fe XVI is also correct, but then both the sulphur and argon abundances are much lower in higher-temperature regions. This second option is favoured here.

If this result is confirmed, it could explain some of the large discrepancies found in the literature by assuming that abundances vary at different heights. For example, observations in the X-rays, associated with hot $(\log T[\mathrm{~K}]=6.5-7.0)$ plasmas often suggest lower "coronal" abundances when compared to observations at lower heights (transition-region temperatures) in the EUV/UV (see, e.g. Veck \& Parkinson 1981), which often produce measurements close to photospheric values. A detailed discussion of these questions is deferred to a future paper.

Interestingly, the sequence of sulphur ions ( $\mathrm{Sx}, \mathrm{S}$ xI, S xII, S XIII) presents the opportunity to measure the sulphur abundance relative to iron quite accurately. The Asplund et al. (2009) recommended photospheric abundance $\mathrm{S} / \mathrm{Fe}$ ratio is 0.42 . The present quiet Sun off-limb spectrum confirms this value. Gloeckler \& Geiss (2007) used Ulysses measurements of solarwind particles emanating from coronal holes and found a similar $\mathrm{S} / \mathrm{Fe}$ ratio (0.52).

A range of values are found in solar flares. One of the most recent and direct line/continuum analyses of RESIK X-ray spectra (Chifor et al. 2007) produced an $\mathrm{S} / \mathrm{Fe}$ ratio of 0.25 , much lower than photospheric. Monsignori Fossi et al. (1994) used a SERTS 1989 rocket spectrum over an active region (on-disk) to obtain the same value, a $\mathrm{S} / \mathrm{Fe}$ ratio of 0.25 . The present is possibly the first measurement of the sulphur abundance in the diffuse AR emission, suggesting the same $\mathrm{S} / \mathrm{Fe}$ ratio of 0.25 , i.e. a first ionization potential (FIP) bias of 1.7.

A similar result was found by Feldman et al. (1998) from off-limb SOHO / SUMER observations of a quiescent streamer. Mohan et al. (2000) used off-limb SOHO / SUMER observations above an active region to find a FIP bias of 2-3 in the S/Si abundance. Brooks \& Warren (2011) used Hinode/EIS observations to find a FIP bias of about 3 in the S/Si abundance in the "coronal outflow" regions, where blueshifts in coronal lines are observed.

The active region spectrum indicates an $\mathrm{Ar} / \mathrm{Fe}$ relative abundance of 0.08, while the quiet Sun one indicates a value of 0.16 . Other nearby regions within the two off-limb observations have been analysed, and the results are the same.

These are the first measurements obtained using Ar XI. There is no measurement of the photospheric abundance of argon, and there is ample literature on the large discrepancies between all previous solar measurements and those obtained from stars 
or nebulae in the solar neighbourhood. Most solar measurements have produced an abundance about a factor of two lower. For some details see, e.g. the recent Lanz et al. (2008) study. Asplund et al. (2009) used some solar measurements to recommend an $\mathrm{Ar} / \mathrm{Fe}$ relative abundance of 0.08. Lanz et al. (2008) recommended instead a higher photospheric value, between 0.11 and 0.17 . There are plenty of solar flare measurements based on the he-like X-ray argon lines, but they all provide low argon abundances (e.g. an Ar/Fe abundance of 0.05, Chifor et al. 2007). There are indications, however, that the argon abundance increases when low-activity X-ray spectra are examined. For example, Sylwester et al. (2010) found $\mathrm{Ar} / \mathrm{Fe}$ values of 0.09 . Monsignori Fossi et al. (1994) measured an $\mathrm{Ar} / \mathrm{Fe}$ abundance of 0.04, from the SERTS 1989 active region rocket spectrum. Doschek \& Bhatia (1990) used an Ar xi forbidden line to measure an Ar/Fe abundance of 0.031 from Skylab S082-B slit spectrograph exposures taken off-limb above an active region.

Argon is a high-FIP element, hence it is expected to show a variation in its abundance depending on the region of the solar atmosphere considered. It is expected that its abundance in active regions should be lower than in the quiet Sun. Indeed, there are measurements far off-limb of very weak argon lines observed by $\mathrm{SOHO} / \mathrm{UVCS}$ that suggest sub-photospheric argon abundances above active regions (Raymond et al. 1997), although most other elements were also found to have sub-photospheric abundances.

The present EIS measurements do indicate that the argon abundance decreases, as does the sulphur one, by a factor of about two from the quiet Sun to active region diffuse emission. The present quiet-Sun measurement remarkably agrees with the value recommended by Lanz et al. (2008), hence if confirmed would end the 40-year long puzzle of the solar photospheric argon abundance.

It is obviously possible that all argon lines are blended with unidentified coronal lines in the Hinode/EIS spectra. If that was the case, the quiet-Sun argon abundance would decrease. However, any blending would still be present in the off-limb active region spectrum, where the argon abundance is already a factor of two lower and is close to the RESIK measurement of Sylwester et al. (2010). A detailed discussion of these questions is deferred to a future paper.

\section{Conclusions}

Spatially-resolved high-resolution EUV solar spectroscopy is very valuable for line identification purposes. After very careful data analysis, Hinode EIS spectra can provide very accurate wavelengths (down to a few $\mathrm{m} \AA$ ) and line intensities. The overall benchmark of the most recent atomic data using offlimb coronal spectra is very satisfactory, at last. All the strongest lines in the spectra have now finally been identified, and agree well (within a relative $20 \%$ or better) in terms of expected and observed line intensities when blending is taken into account. Indeed, the majority of spectral lines are blended. Only blending with coronal lines was taken into account here. Some wavelengths have been revised.

A large number of transitions still await firm identifications, however, the results presented here clearly indicate the approximate formation temperature of the unidentified lines, which will aid the identification process. Work is in progress to improve some of the atomic models for a range of ions, but new laboratory measurements are essential to confirm the many tentative identifications provided here.

A few important temperature diagnostics for Hinode/EIS were discussed in Del Zanna (2009a,b and 2010) and have not been repeated here. The various calibration problems for the Hinode/EIS instrument, highlighted here, will need to be properly assessed before these new temperature diagnostics can be used reliably. The main density diagnostics for Hinode/EIS have now been assessed. Very good agreement from different ions is found, which supports the accuracy of the new atomic data.

The results of the emission measure analysis are new and potentially important, if confirmed. A quiet-Sun argon abundance in line with Galactic measurements is found. Argon and sulphur show the well-know FIP bias, i.e. their abundances (relative to iron) are much reduced (by a factor of about two) in active region diffuse emission when compared to the quiet Sun. The emission measures at high temperatures $(\log T[\mathrm{~K}]=6.4-6.7)$ suggest that the argon and sulphur FIP bias might increase even more with temperature. These questions will be discussed in a forthcoming paper.

Acknowledgements. Support from STFC (Advanced Fellowship and APAP network) is acknowledged.

The work of the UK APAP Network was funded by the UK STFC under grant No. PP/E001254/1 with the University of Strathclyde. The anonymous referee is thanked for useful suggestions. I warmly thank B. C. Fawcett for his encouragement and help since 2003, when the present long-term benchmark programme started. CHIANTI is a collaborative project involving researchers at the Universities of: Cambridge (UK), George Mason, Michigan (USA). The excellent Hinode Science Data Centre Europe was used to search the EIS database. Hinode is a Japanese mission developed and launched by ISAS/JAXA, with NAOJ as domestic partner and NASA and STFC (UK) as international partners. It is operated by these agencies in co-operation with ESA and NSC (Norway).

\section{References}

Asplund, M., Grevesse, N., Sauval, A. J., \& Scott, P. 2009, ARA\&A, 47, 481 Behring, W. E., Cohen, L., Doschek, G. A., \& Feldman, U. 1976, ApJ, 203, 521 Brooks, D. H., \& Warren, H. P. 2011, ApJ, 727, L13

Brown, C. M., Feldman, U., Seely, J. F., Korendyke, C. M., \& Hara, H. 2008, ApJS, 176, 511

Chifor, C., Del Zanna, G., Mason, H. E., et al. 2007, A\&A, 462, 323

Culhane, J. L., Harra, L. K., James, A. M., et al. 2007, Sol. Phys., 60

Del Zanna, G. 2008, A\&A, 481, L69

Del Zanna, G. 2009a, A\&A, 508, 501

Del Zanna, G. 2009b, A\&A, 508, 513

Del Zanna, G. 2010, A\&A, 514, A41

Del Zanna, G. 2011, A\&A, 533, A12

Del Zanna, G., \& Ishikawa, Y. 2009, A\&A, 508, 1517

Del Zanna, G., \& Mason, H. E. 2003, A\&A, 406, 1089

Del Zanna, G., \& Mason, H. E. 2005, A\&A, 433, 731

Del Zanna, G., Landini, M., \& Mason, H. E. 2002, A\&A, 385, 968

Del Zanna, G., Berrington, K. A., \& Mason, H. E. 2004, A\&A, 422, 731

Del Zanna, G., Storey, P. J., \& Mason, H. E. 2010, A\&A, 514, A40

Del Zanna, G., O’ Dwyer, B., \& Mason, H. E. 2011, A\&A, 535, A46

Dere, K. P., Landi, E., Mason, H. E., Monsignori Fossi, B. C., \& Young, P. R. 1997, A\&AS, 125, 149

Dere, K. P., Landi, E., Young, P. R., et al. 2009, A\&A, 498, 915

Doschek, G. A., \& Bhatia, A. K. 1990, ApJ, 358, 338

Feldman, U., Schühle, U., Widing, K. G., \& Laming, J. M. 1998, ApJ, 505, 999

Gloeckler, G., \& Geiss, J. 2007, Space Sci. Rev., 130, 139

Haugan, S. V. H. 1997, CDS software note, 47

Landi, E., \& Young, P. R. 2009, ApJ, 706, 1

Landi, E., Del Zanna, G., Young, P. R., Dere, K. P., \& Mason, H. E. 2011, ApJ, accepted

Lang, J., Kent, B. J., Paustian, W., et al. 2006, Appl. Opt., 45, 8689

Lanz, T., Cunha, K., Holtzman, J., \& Hubeny, I. 2008, ApJ, 678, 1342

Liang, G. Y., \& Badnell, N. R. 2010, A\&A, 518, A64

Liang, G. Y., Whiteford, A. D., \& Badnell, N. R. 2009, A\&A, 499, 943

Liang, G. Y., Badnell, N. R., Crespo López-Urrutia, J. R., et al. 2010, ApJS, 190, 322

Malinovsky, L., \& Heroux, M. 1973, ApJ, 181, 1009

Mohan, A., Landi, E., \& Dwivedi, B. N. 2000, A\&A, 364, 835

Monsignori Fossi, B. C., Landini, M., Thomas, R. J., \& Neupert, W. M. 1994, Adv. Space Res., 14, 163

O’Dwyer, B., Del Zanna, G., Mason, H. E., Weber, M. A., \& Tripathi, D. 2010, A\&A, 521, A21

Raymond, J. C., Kohl, J. L., Noci, G., et al. 1997, Sol. Phys., 175, 645 
G. Del Zanna: Benchmarking atomic data for the CHIANTI atomic database: coronal lines observed by Hinode EIS

Sampson, D. H., Zhang, H. L., \& Fontes, C. J. 1990, Atomic Data and Nuclear Data Tables, 44, 209

Storey, P. J., \& Zeippen, C. J. 2010, A\&A, 511, A78

Storey, P. J., Mason, H. E., \& Young, P. R. 2000, A\&AS, 141, 285

Storey, P. J., Zeippen, C. J., \& Le Dourneuf, M. 2002, A\&A, 394, 753

Storey, P. J., Del Zanna, G., Mason, H. E., \& Zeippen, C. 2005, A\&A, 433, 717

Sylwester, B., Sylwester, J., \& Phillips, K. J. H. 2010, A\&A, 514, A82

Veck, N. J., \& Parkinson, J. H. 1981, MNRAS, 197, 41

Witthoeft, M. C., \& Badnell, N. R. 2008, A\&A, 481, 543

Young, P. R. 2009, ApJ, 691, L77

Young, P. R., Del Zanna, G., Mason, H. E., et al. 2007, PASJ, 59, 857

\section{Appendix A: The quiet-Sun off-limb spectrum}

Figure A.1 shows the Hinode EIS spectrum of the quiet-Sun offlimb region observed on 2007 March 11. Table A.1 lists a selection of the main lines in this spectrum. Figure A.2 shows how line intensities vary across the limb, to aid the identification process. Figures A.3-A.7 show the emissivity ratio curves relative to the Fe x,Fe xI,Fe xII, Fe xIII, Fe xIV and Si x for the quiet-Sun off-limb region observed on 2007 March 11.
Table A.1. List of emission lines from the 2007 March 11 off-limb quiet-Sun spectrum.

\begin{tabular}{|c|c|c|c|c|c|}
\hline$\lambda_{\mathrm{o}}$ & DN & Int & $F$ & ID & $\lambda_{\mathrm{r}}$ \\
\hline 171.074 & 10.5 & 140.7 & 68 & $\mathrm{Fe}$ IX & 171.072 \\
\hline 174.535 & 53.3 & 119.7 & 69 & $\mathrm{Fe} x$ & 174.534 \\
\hline 177.241 & 87.8 & 65.8 & 69 & Fex (bl Fe VII) & 177.242 \\
\hline 178.065 & 12.3 & 6.5 & 75 & $\mathrm{Fe} \mathrm{XI}$ & 178.056 \\
\hline 179.756 & 6.9 & 1.9 & 65 & Fe XI & 179.760 \\
\hline 180.403 & 608.1 & 133.6 & 73 & Fe xi (bl Fe X, u TR) & 180.400 \\
\hline 180.594 & 24.2 & 5.0 & 77 & $\mathrm{Fe} \mathrm{XI}$ & 180.595 \\
\hline 180.750 & 7.4 & 1.4 & 77 & $\mathrm{Sx}$ & 180.730 \\
\hline 181.135 & 32.5 & 5.5 & 66 & Fe XI (bl oc u TR) & 181.131 \\
\hline 182.168 & 177.9 & 21.3 & 69 & $\mathrm{Fe} \mathrm{XI}$ & 182.167 \\
\hline 182.303 & 26.9 & 3.1 & 77 & Fe $x(b l u T R)$ & 182.308 \\
\hline 183.944 & 18.8 & 1.3 & 77 & $\mathrm{O}_{\mathrm{VI}}$ & 183.937 \\
\hline 184.119 & 29.8 & 1.9 & 77 & O vi (bl Ni VIII?) & 184.117 \\
\hline 184.413 & 33.2 & 2.0 & 77 & u X (bl Fe xI) & 184.420 \\
\hline 184.538 & 579.7 & 33.6 & 70 & Fe x (bl u ?) & 184.543 \\
\hline 184.689 & 17.4 & 1.0 & 77 & u X & \\
\hline 184.800 & 36.5 & 2.0 & 66 & Fe XI (bl) & 184.793 \\
\hline 185.210 & 61.1 & 3.0 & 85 & Fe vIII (bl oc Ni XVI) & 185.216 \\
\hline 185.457 & 9.1 & 0.4 & 77 & ? Mn VIII & 185.460 \\
\hline 185.564 & 10.0 & 0.5 & 71 & u (bl oc Fe viI) & \\
\hline 185.619 & 9.5 & 0.4 & 65 & $\mathrm{u}$ & \\
\hline 185.711 & 17.4 & 0.7 & 77 & $\mathrm{u}$ & \\
\hline 186.000 & 12.5 & 0.5 & 77 & $\mathrm{u}$ & \\
\hline 186.117 & 14.3 & 0.6 & 77 & $\mathrm{u}$ & \\
\hline 186.249 & 27.5 & 1.1 & 77 & Fe XII & 186.248 \\
\hline 186.609 & 70.4 & 2.5 & 85 & Fe vIII (bl oc Ca xIv) & 186.605 \\
\hline 186.876 & 610.4 & 20.5 & 87 & Fe xII (sbl, bl S xI) & 186.880 \\
\hline 186.989 & 92.1 & 3.0 & 65 & Fe XII (bl u) & 187.000 \\
\hline 187.101 & 29.9 & 1.0 & 77 & $? \mathrm{Mg}$ x (bl Ar xI?) & \\
\hline 187.208 & 21.9 & 0.7 & 77 & ? Mg x (bl Fe VIII) & 187.240 \\
\hline 187.437 & 26.6 & 0.8 & 77 & $\mathrm{Fe} \mathrm{xI}$ & 187.450 \\
\hline 187.579 & 20.8 & 0.6 & 77 & $\mathrm{u}$ & \\
\hline 187.681 & 20.5 & 0.6 & 77 & $\mathrm{u}$ & \\
\hline 187.847 & 25.8 & 0.7 & 65 & $\mathrm{u}$ & \\
\hline 187.951 & 55.9 & 1.5 & 77 & u VIII-IX (bl oc Ar xIV) & \\
\hline 188.216 & 3111.1 & 81.5 & 73 & Fe xI (bl oc Fe VII) & 188.216 \\
\hline 188.299 & 1919.1 & 49.6 & 65 & $\mathrm{Fe} \mathrm{XI}$ & 188.299 \\
\hline 188.367 & 192.9 & 4.9 & 77 & $\mathrm{u}$ & \\
\hline 188.490 & 247.3 & 6.2 & 73 & Fe Ix (bl Mn IX ?) & 188.490 \\
\hline 188.579 & 28.8 & 0.7 & 69 & u VIII (Ni vIII?) & 188.570 \\
\hline 188.667 & 131.3 & 3.2 & 77 & S xi (bl Fe IX) & 188.667 \\
\hline 188.805 & 96.4 & 2.3 & 77 & Ar XI & 188.82 \\
\hline 189.007 & 96.2 & 2.2 & 77 & Fe XI & 189.020 \\
\hline 189.121 & 193.6 & 4.4 & 77 & Fe xI (bl u) & 189.123 \\
\hline 189.254 & 29.2 & 0.7 & 77 & $\mathrm{u}$ & 189.240 \\
\hline 189.407 & 33.3 & 0.7 & 77 & u (bl oc Fe viI 189.450) & \\
\hline 189.585 & 30.6 & 0.6 & 77 & $\operatorname{Ar} \mathrm{XI}(\mathrm{bl} \mathrm{u})$ & 189.57 \\
\hline 189.724 & 156.6 & 3.3 & 77 & Fe XI & 189.733 \\
\hline 189.929 & 154.7 & 3.1 & 73 & Fe IX & 189.940 \\
\hline 190.038 & 728.4 & 14.6 & 77 & Fe x (bl Fe xI) & 190.038 \\
\hline 190.320 & 77.0 & 1.5 & 77 & u XII & \\
\hline 190.386 & 185.6 & 3.5 & 69 & Fe xI (bl u) & 190.390 \\
\hline 190.508 & 19.1 & 0.4 & 77 & $\mathrm{u}$ & \\
\hline 190.703 & 25.6 & 0.5 & 77 & $\mathrm{u}$ & \\
\hline 190.877 & 61.6 & 1.1 & 77 & $? \operatorname{Nax}$ & \\
\hline 191.043 & 115.8 & 2.0 & 77 & Fe XII (bl Ar XI) & 191.050 \\
\hline 191.255 & 260.5 & 4.5 & 77 & Fe Ix (bl S xI) & 191.210 \\
\hline 191.406 & 44.8 & 0.8 & 77 & u (bl oc Ar XIV) & \\
\hline 191.583 & 37.3 & 0.6 & 79 & $\mathrm{u}$ & \\
\hline 191.710 & 34.7 & 0.6 & 79 & $\mathrm{u}$ & \\
\hline 191.845 & 54.5 & 0.9 & 79 & $\mathrm{u}$ & \\
\hline 192.019 & 192.6 & 3.1 & 79 & Fe xI (bl oc many) & 192.020 \\
\hline 192.095 & 36.1 & 0.6 & 65 & u X & \\
\hline 192.181 & 139.7 & 2.2 & 79 & Fe XII & $\mathrm{TN}$ \\
\hline 192.291 & 67.2 & 1.0 & 65 & u X & \\
\hline 192.394 & 2865.0 & 44.4 & 73 & Fe XII & 192.390 \\
\hline 192.622 & 167.1 & 2.5 & 79 & $\mathrm{Fe}(\mathrm{xI}(\mathrm{bl} \mathrm{Ni} \mathrm{x} ?)$ & 192.630 \\
\hline 192.811 & 1439.5 & 21.6 & 72 & Fe xI (bl u, oc Ca xvII, Ov) & 192.813 \\
\hline 192.880 & 62.0 & 0.9 & 79 & $\mathrm{Ov}(\mathrm{bl} ?)$ & 192.910 \\
\hline 193.204 & 48.5 & 0.7 & 79 & $\mathrm{u}$ & \\
\hline 193.347 & 70.8 & 1.0 & 79 & u VII-VIII & \\
\hline 193.508 & 6636.0 & 94.6 & 74 & Fe XII & 193.509 \\
\hline 193.706 & 292.7 & 4.1 & 79 & $\mathrm{Fe} \times(\mathrm{bl} \mathrm{u})$ & 193.715 \\
\hline 193.837 & 42.5 & 0.6 & 77 & Ca XIV (bl u) & 193.866 \\
\hline 193.951 & 31.0 & 0.4 & 79 & u (bl oc Fe vIII) & 193.968 \\
\hline 194.073 & 61.6 & 0.8 & 79 & Ar XI & 194.09 \\
\hline 194.315 & 116.6 & 1.6 & 79 & u IX-X & \\
\hline 194.447 & 35.3 & 0.5 & 79 & u (bl oc Ar XIV) & \\
\hline 194.637 & 90.9 & 1.2 & 79 & Fe VIII & 194.660 \\
\hline 194.817 & 80.1 & 1.1 & 79 & $\mathrm{u}$ & \\
\hline 195.117 & 10257.5 & 136.2 & 74 & Fe XII (sbl) & 195.119 \\
\hline
\end{tabular}


Table A.1. continued.

\begin{tabular}{|c|c|c|c|c|c|}
\hline$\lambda_{\mathrm{o}}$ & DN & Int & $F$ & ID & $\lambda_{\mathrm{r}}$ \\
\hline 195.239 & 165.9 & 2.2 & 79 & u XI-XII (bl Ni XvI) & \\
\hline 195.388 & 191.7 & 2.5 & 79 & $\mathrm{uX}$ & 195.390 \\
\hline 195.509 & 66.5 & 0.9 & 79 & $\mathrm{u}$ & \\
\hline 195.724 & 49.8 & 0.7 & 79 & $\mathrm{u}$ & \\
\hline 195.952 & 42.8 & 0.6 & 79 & Fe VIII & 195.972 \\
\hline 196.097 & 35.5 & 0.5 & 79 & ? Fe VII & 196.046 \\
\hline 196.263 & 31.1 & 0.4 & 79 & ? Fe VII (sbl) & 196.210 \\
\hline 196.391 & 33.4 & 0.4 & 79 & $\mathrm{u}$ & \\
\hline 196.519 & 170.3 & 2.3 & 74 & Fe XIII & 196.525 \\
\hline 196.644 & 490.6 & 6.6 & 78 & Fe xII (bl u, oc Fe vIII) & 196.640 \\
\hline 196.814 & 69.1 & 0.9 & 79 & $\mathrm{u}$ & \\
\hline 196.912 & 65.2 & 0.9 & 75 & $\mathrm{u}$ & \\
\hline 197.021 & 72.1 & 1.0 & 79 & u XIV & \\
\hline 197.347 & 38.3 & 0.5 & 79 & u (bl Fe vIII,Fe vII & 197.362 \\
\hline 197.427 & 242.1 & 3.4 & 79 & Fe xIII (bl Ni x?) & 197.433 \\
\hline 197.598 & 36.9 & 0.5 & 79 & Nix & \\
\hline 197.856 & 267.7 & 4.0 & 79 & $\mathrm{Fe}_{\mathrm{IX}}$ & 197.850 \\
\hline 198.054 & 37.7 & 0.6 & 79 & $\mathrm{u}$ & 198.050 \\
\hline 198.287 & 29.8 & 0.5 & 79 & $\mathrm{u}$ & \\
\hline 198.424 & 106.6 & 1.7 & 75 & u XI (bl Ni xI) & \\
\hline 198.550 & 492.8 & 8.2 & 79 & S VIII (bl Fe xI) & 198.550 \\
\hline 198.750 & 48.1 & 0.8 & 79 & $\mathrm{u}$ & \\
\hline 198.951 & 44.7 & 0.8 & 79 & $\mathrm{u}$ & \\
\hline 199.237 & 51.3 & 1.0 & 79 & $\mathrm{u}$ & \\
\hline 199.336 & 19.9 & 0.4 & 79 & $\mathrm{u}$ & \\
\hline 199.600 & 52.1 & 1.1 & 79 & $\mathrm{u}$ & \\
\hline 199.816 & 56.0 & 1.3 & 79 & u XI (bl oc Ni XVII) & \\
\hline 200.009 & 302.8 & 7.2 & 89 & Fe XIII & 200.021 \\
\hline 200.169 & 27.2 & 0.7 & 79 & $\mathrm{u}$ & \\
\hline 200.341 & 49.2 & 1.3 & 79 & u XI-XII & \\
\hline 200.441 & 23.8 & 0.6 & 79 & $\mathrm{u}$ & \\
\hline 200.684 & 83.7 & 2.5 & 79 & u X-XII & \\
\hline 200.798 & 28.5 & 0.9 & 79 & $\mathrm{u}$ & \\
\hline 200.998 & 26.8 & 0.9 & 79 & $\mathrm{Caxv}(\mathrm{bl})$ & 200.980 \\
\hline 201.117 & 796.8 & 27.8 & 85 & Fe xIII (bl) & 201.121 \\
\hline 201.498 & 86.3 & 3.5 & 79 & $\mathrm{u}$ & \\
\hline 201.573 & 103.1 & 4.2 & 79 & Fe XIII (bl) & \\
\hline 201.738 & 156.5 & 7.0 & 79 & Fe XI (bl oc u) & 201.734 \\
\hline 201.918 & 29.5 & 1.4 & 79 & u (bl oc Fe vII) & 201.860 \\
\hline 202.046 & 2043.1 & 103.3 & 74 & Fe XIII & 202.044 \\
\hline 202.425 & 221.1 & 13.0 & 79 & $\mathrm{Fe} \times \mathrm{xI}(\mathrm{bl} \mathrm{u})$ & 202.420 \\
\hline 202.603 & 78.5 & 5.0 & 79 & S VIII (bl Fe XI) & 202.608 \\
\hline 202.710 & 141.0 & 9.3 & 74 & Fe xi (bl Fe xIII) & 202.710 \\
\hline 202.892 & 17.0 & 1.2 & 79 & $\mathrm{u}$ & \\
\hline 203.166 & 53.1 & 4.1 & 79 & Fe xIII (bl Fe xII) & 203.165 \\
\hline 203.722 & 131.5 & 12.3 & 78 & Fe xII (bl Fe xIII) & 203.728 \\
\hline 203.822 & 243.1 & 23.4 & 88 & Fe XIII (sbl) & 203.826 \\
\hline 204.254 & 39.0 & 4.2 & 79 & Fe xIII & 204.263 \\
\hline 204.351 & 17.7 & 2.0 & 79 & u IX-XII & \\
\hline 204.730 & 12.8 & 1.5 & 79 & u VIII (bl oc Fe viII) & 204.700 \\
\hline 204.937 & 57.3 & 7.3 & 79 & Fe xIII & 204.942 \\
\hline 206.173 & 31.8 & 5.2 & 79 & $\mathrm{Fe} \mathrm{xI}(\mathrm{bl} \mathrm{u})$ & 206.170 \\
\hline 206.259 & 32.1 & 5.3 & 65 & Fe XI (bl u) & 206.250 \\
\hline 206.365 & 29.6 & 5.0 & 79 & Fe xII (bl u) & 206.371 \\
\hline 207.449 & 35.6 & 7.3 & 77 & $\mathrm{Fe} \times(\mathrm{bl} \mathrm{u})$ & 207.449 \\
\hline 207.747 & 13.6 & 2.9 & 79 & Fe xI (bl u) & 207.712 \\
\hline 207.946 & 34.7 & 7.8 & 79 & u XI (bl Ni XI ?) & \\
\hline 208.310 & 12.8 & 3.1 & 79 & Fe xiI (bl Ca xv, u) & 208.318 \\
\hline 209.108 & 15.7 & 4.4 & 79 & Fe XII $(\mathrm{bl} \mathrm{u})$ & \\
\hline 209.620 & 16.0 & 4.9 & 73 & Fe XIII (bl u) & 209.617 \\
\hline 209.766 & 15.3 & 4.8 & 79 & $\mathrm{Fe} \mathrm{xI}(\mathrm{bl} \mathrm{u})$ & \\
\hline 209.920 & 51.3 & 16.7 & 77 & Fe xIII (bl oc u TR) & 209.919 \\
\hline 211.320 & 80.6 & 33.4 & 73 & Fe xIV (bl) & 211.316 \\
\hline 211.442 & 12.3 & 5.2 & 79 & u XII (bl Ni xI) & \\
\hline 246.211 & 50.2 & 10.3 & 76 & Fe XIII (bl oc Si vi) & 246.208 \\
\hline 246.913 & 10.4 & 2.0 & 77 & $\mathrm{u}$ & \\
\hline 247.168 & 12.1 & 2.3 & 75 & u (bl S xi Fe xII) & 247.147 \\
\hline 247.405 & 16.8 & 3.2 & 76 & $\mathrm{u}$ & \\
\hline 248.502 & 14.7 & 2.6 & 77 & $\mathrm{u}(\mathrm{bl} \mathrm{oc} \mathrm{Ov})$ & 248.490 \\
\hline 249.383 & 35.9 & 6.1 & 77 & Fe xII (bl u) & 249.388 \\
\hline 251.872 & 20.0 & 2.7 & 75 & Fe xII(bl u) & \\
\hline 251.952 & 143.7 & 19.2 & 79 & Fe XIII & 251.953 \\
\hline 252.199 & 19.2 & 2.5 & 79 & Fe xiv & 252.197 \\
\hline 253.793 & 72.1 & 8.2 & 83 & Si $x$ & 253.795 \\
\hline 254.598 & 54.6 & 5.8 & 72 & $\mathrm{Fe} \mathrm{xI}$ & \\
\hline 256.280 & 96.4 & 9.1 & 80 & $\mathrm{He}_{\text {II }}(\mathrm{bl})$ & 256.320 \\
\hline 256.384 & 435.3 & 40.8 & 109 & Si x (bl) & 256.400 \\
\hline 256.659 & 10.2 & 0.9 & 79 & S XIII & 256.686 \\
\hline 256.926 & 233.5 & 21.1 & 79 & Fe xI (bl Fe xII) & 256.925 \\
\hline 257.150 & 87.8 & 7.8 & 79 & $\mathrm{Sx}$ & 257.150 \\
\hline 257.264 & 463.9 & 41.1 & 73 & $\mathrm{Fe} x$ & 257.262 \\
\hline 257.393 & 55.0 & 4.9 & 79 & Fe xiv & 257.300 \\
\hline
\end{tabular}

Table A.1. continued.

\begin{tabular}{|c|c|c|c|c|c|}
\hline$\lambda_{\mathrm{o}}$ & DN & Int & $F$ & ID & $\lambda_{\mathrm{r}}$ \\
\hline 257.550 & 141.8 & 12.3 & 79 & Fe XI (sbl) & 257.547 \\
\hline 257.771 & 72.2 & 6.2 & 79 & Fe xI (bl u) & 257.772 \\
\hline 257.913 & 27.1 & 2.3 & 79 & $\mathrm{u}$ & \\
\hline 258.084 & 30.9 & 2.6 & 79 & ? Si Ix & 258.082 \\
\hline 258.376 & 535.8 & 44.1 & 84 & Si x & 258.372 \\
\hline 259.496 & 228.6 & 17.6 & 88 & $\mathrm{~S} x$ (bl Fe XII) & 259.494 \\
\hline 259.975 & 40.9 & 3.1 & 76 & Fe XII (bl u) & \\
\hline 261.059 & 375.4 & 26.3 & 84 & Si $\mathrm{x}$ & 261.056 \\
\hline 261.432 & 10.0 & 0.7 & 79 & $\mathrm{u}$ & \\
\hline 261.741 & 40.6 & 2.7 & 79 & Fe XIII & 261.730 \\
\hline 262.975 & 5.7 & 0.4 & 79 & Fe xVI (bl Fe XIII) & 262.984 \\
\hline 263.452 & 6.9 & 0.4 & 79 & $\mathrm{u}$ & \\
\hline 264.235 & 386.9 & 22.9 & 85 & $\mathrm{~S} \times(\mathrm{bl}$ oc u) & 264.233 \\
\hline 264.785 & 296.5 & 17.1 & 80 & Fe xIV (bl Fe xI) & 264.787 \\
\hline 264.912 & 6.5 & 0.4 & 79 & Fe xVI & 265.000 \\
\hline 265.796 & 7.2 & 0.4 & 79 & $\mathrm{u}$ & \\
\hline 266.085 & 23.7 & 1.3 & 79 & $\mathrm{u}$ & \\
\hline 266.568 & 22.4 & 1.2 & 70 & $\mathrm{u}$ & \\
\hline 266.619 & 28.7 & 1.5 & 70 & $\mathrm{u}$ & \\
\hline 266.755 & 28.8 & 1.5 & 79 & $\mathrm{u}$ & \\
\hline 267.801 & 18.8 & 1.0 & 119 & ? Ni XI & \\
\hline 269.490 & 12.4 & 0.6 & 79 & $\mathrm{u}$ & \\
\hline 269.871 & 11.3 & 0.6 & 79 & $\mathrm{u}$ & \\
\hline 270.426 & 10.8 & 0.5 & 79 & ? Mg vI & 270.391 \\
\hline 270.519 & 128.2 & 6.4 & 68 & Fe xIV (bl oc) & 270.524 \\
\hline 270.600 & 11.6 & 0.6 & 79 & $\mathrm{u}$ & \\
\hline 271.782 & 14.3 & 0.7 & 79 & $\mathrm{u}$ & \\
\hline 271.991 & 475.0 & 24.3 & 88 & Six & 271.992 \\
\hline 272.156 & 19.1 & 1.0 & 79 & $\mathrm{u}$ & \\
\hline 272.546 & 15.7 & 0.8 & 79 & $\mathrm{u}(\mathrm{bl} F \mathrm{Fe}$ XI) & \\
\hline 272.659 & 13.7 & 0.7 & 79 & Si VII & 272.660 \\
\hline 272.885 & 12.2 & 0.6 & 79 & $\mathrm{u}$ & \\
\hline 273.170 & 9.7 & 0.5 & 79 & $\mathrm{u}$ & \\
\hline 274.203 & 349.1 & 19.4 & 84 & Fe xIV (bl Si VII) & 274.203 \\
\hline 275.367 & 32.8 & 2.0 & 79 & Si VII & 275.35 \\
\hline 275.695 & 9.9 & 0.6 & 79 & Si VII (bl u) & 275.690 \\
\hline 276.056 & 6.5 & 0.4 & 79 & $\mathrm{u}$ & \\
\hline 276.467 & 8.6 & 0.6 & 79 & $\mathrm{u}$ & \\
\hline 276.855 & 27.0 & 1.8 & 79 & Si vIII bl Si VII & 276.839 \\
\hline 277.049 & 74.9 & 5.0 & 115 & Si VIII (bl Mg VII) & 277.000 \\
\hline 277.262 & 273.0 & 18.8 & 88 & Si $\mathrm{x}$ & 277.265 \\
\hline 278.156 & 6.8 & 0.5 & 79 & Si x (bl u) & 278.180 \\
\hline 278.411 & 27.8 & 2.1 & 149 & Mg VII (bl Si viI) & 278.395 \\
\hline 278.648 & 6.3 & 0.5 & 79 & ? Ni XI & \\
\hline 279.479 & 6.5 & 0.6 & 79 & $\mathrm{u}$ & 279.480 \\
\hline 280.129 & 12.0 & 1.1 & 148 & $\mathrm{Al}$ IX (bl u oc $\mathrm{Fe}$ XVII) & 280.160 \\
\hline 281.414 & 46.5 & 4.9 & 79 & $\mathrm{~S} \mathrm{XI}$ & 281.401 \\
\hline 282.422 & 14.7 & 1.7 & 79 & $\mathrm{Al}$ IX & 282.422 \\
\hline 282.980 & 9.5 & 1.2 & 79 & $\mathrm{u}$ & 282.980 \\
\hline 283.465 & 12.0 & 1.6 & 79 & Fe xII (bl u) & \\
\hline 284.029 & 27.1 & 3.9 & 79 & Al Ix (bl oc Fe xvII) & 284.042 \\
\hline 284.161 & 168.6 & 24.3 & 84 & Fexv (bl) & 284.160 \\
\hline 284.614 & 6.0 & 0.9 & 79 & $\mathrm{u}$ & 284.700 \\
\hline 285.602 & 13.8 & 2.3 & 79 & S XI & 285.600 \\
\hline 285.833 & 17.6 & 3.1 & 96 & S XI & 285.828 \\
\hline 286.379 & 5.1 & 0.9 & 79 & $\mathrm{Al}$ IX & 286.380 \\
\hline 288.409 & 6.9 & 1.6 & 98 & S XII & 288.400 \\
\hline 289.146 & 5.1 & 1.3 & 79 & Fe xIV & 289.148 \\
\hline 290.710 & 17.3 & 5.3 & 83 & Si Ix (bl oc Fe vII) & 290.687 \\
\hline 291.018 & 10.3 & 3.3 & 65 & Fe xII & 291.010 \\
\hline
\end{tabular}

Notes. $\lambda_{0}(\AA)$ is the measured wavelength. DN is the number of total counts in the line, while Int is the calibrated intensity (phot $\mathrm{cm}^{-2} \mathrm{~s}^{-1} \operatorname{arcsec}^{-2}$ ). $F$ is the full-width-half-maximum in $\mathrm{m} \AA$, while the column ID provides the identification (bl: blended; sbl: selfblend; u: unidentified; bl oc: blended in other plasma conditions). In a few cases, the class of a line is given (i.e.: u VII is a line with a morphology similar to Fe VII), as well as the rest wavelength $\left(\lambda_{\mathrm{r}}, \AA\right)$ from the literature. " $\mathrm{N}$ " indicates a new identification proposed here, while "T N" a tentative new one. 
G. Del Zanna: Benchmarking atomic data for the CHIANTI atomic database: coronal lines observed by Hinode EIS
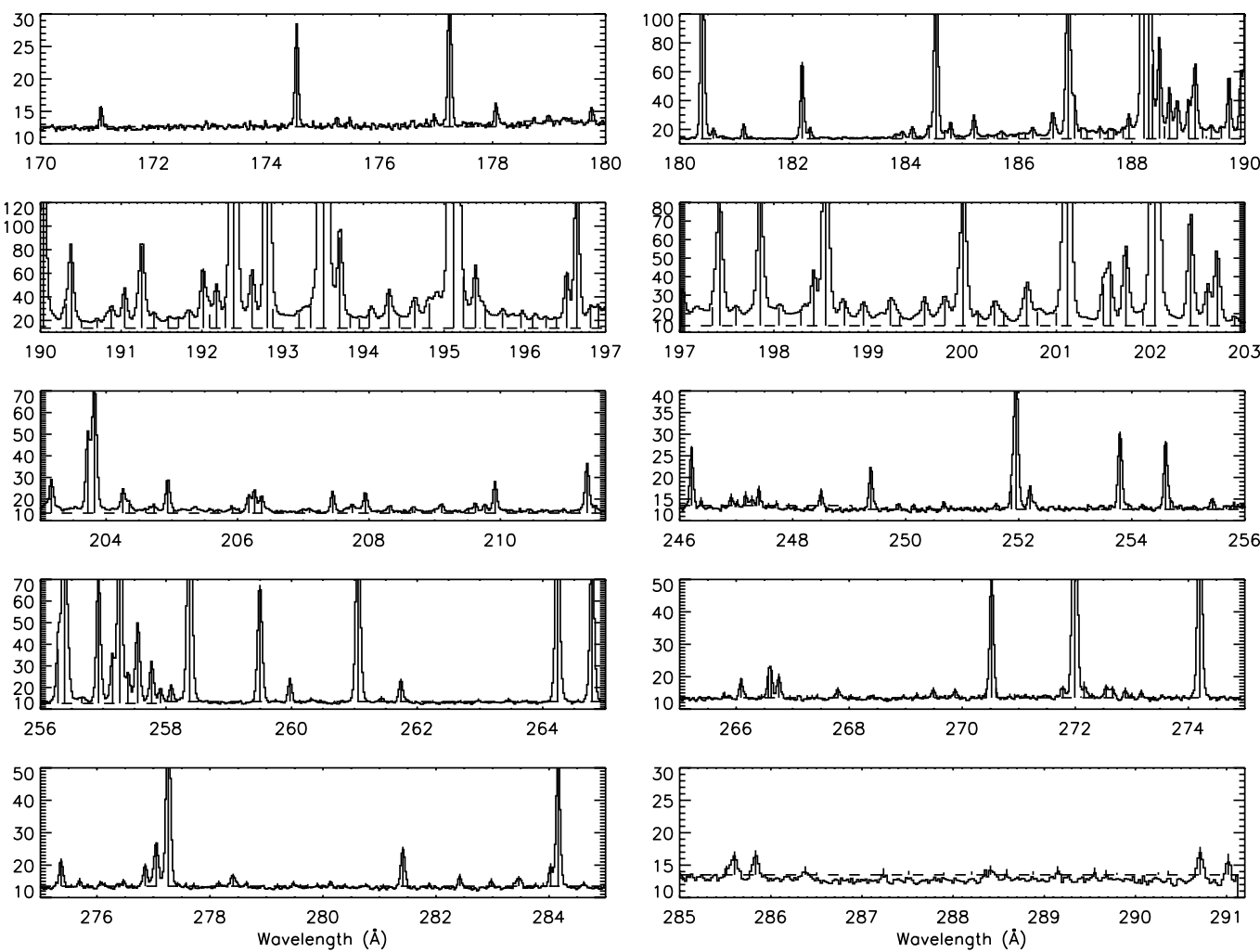

Fig. A.1. Hinode EIS spectrum (units are averaged counts per pixel) of the quiet-Sun off-limb region observed on 2007 March 11 (see Fig. 4).
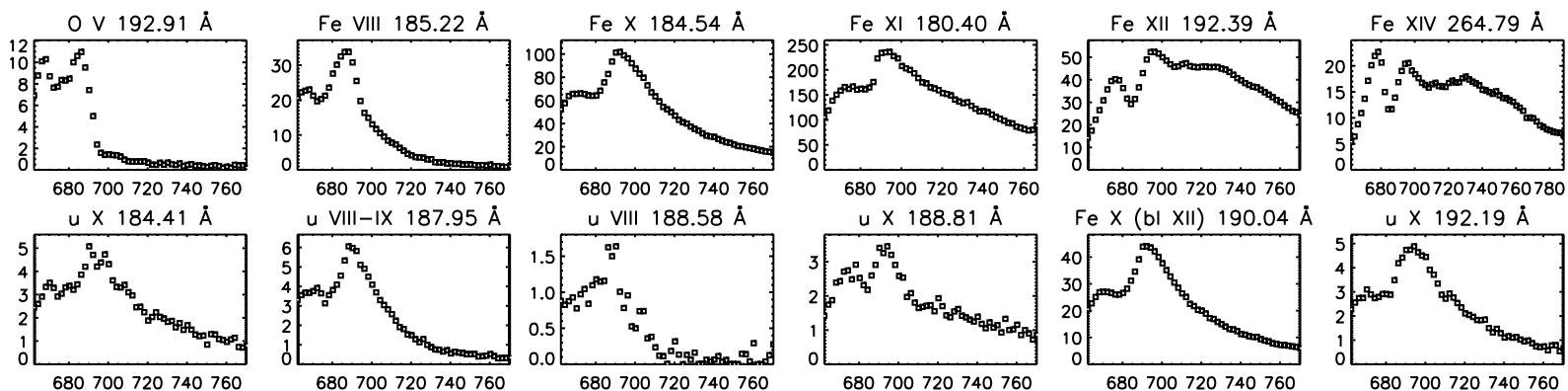

$u \times 188.81 \AA$

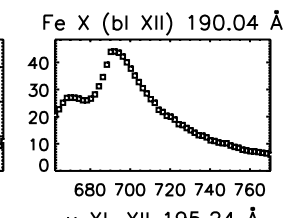

u X $192.19 \AA$
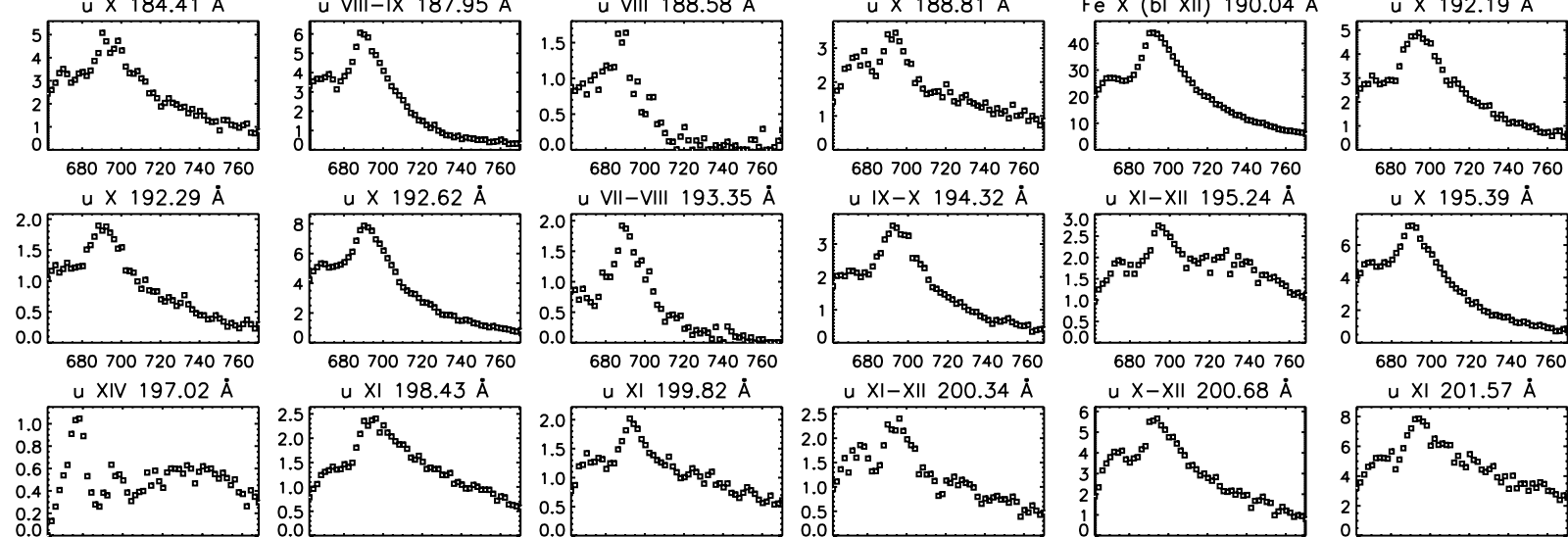

680700720740760
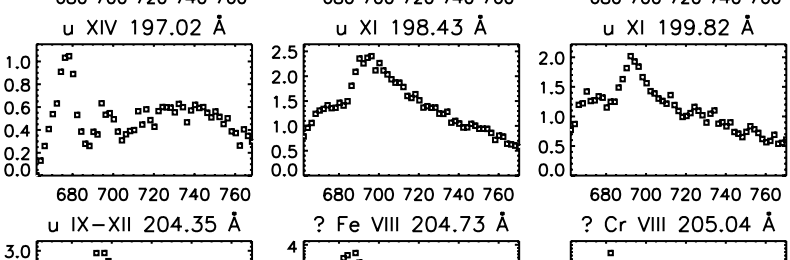

? Fe VIII 204.7

680700720740760
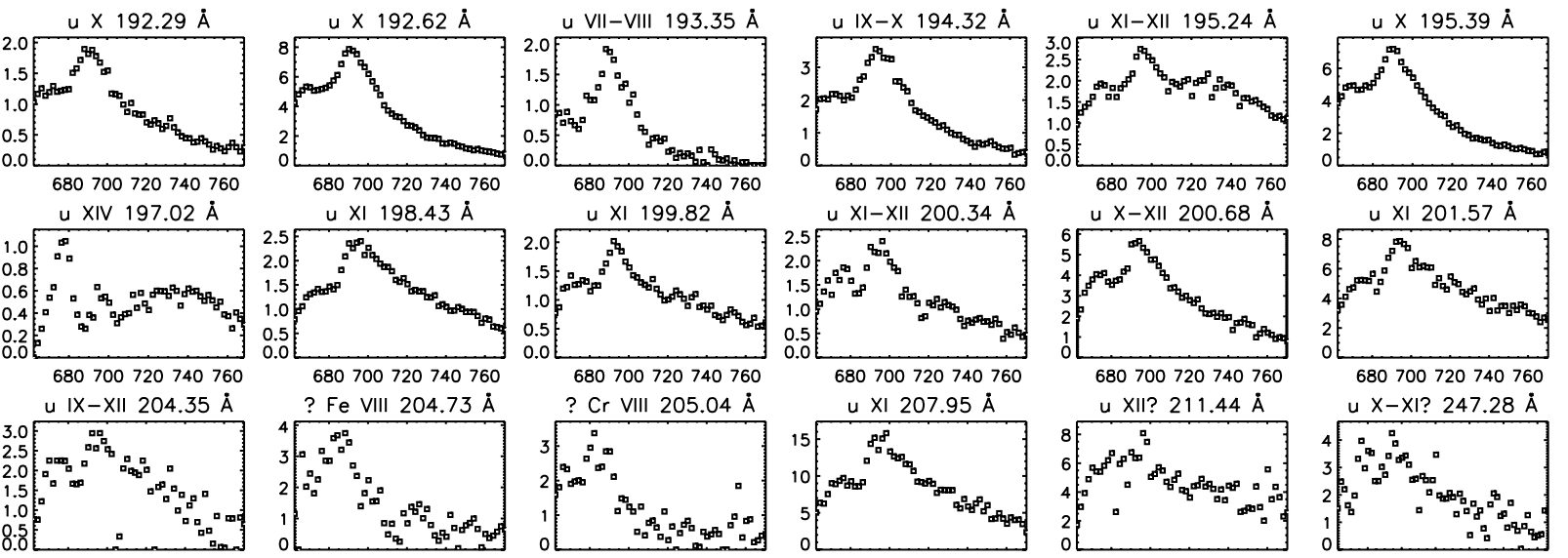

680700720740760
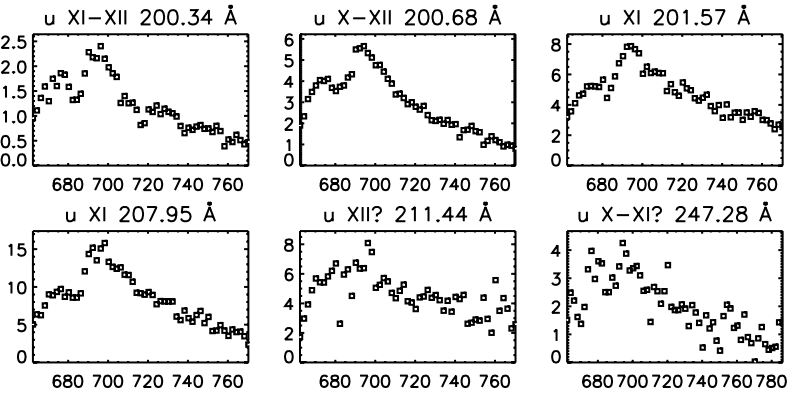

680700720740760
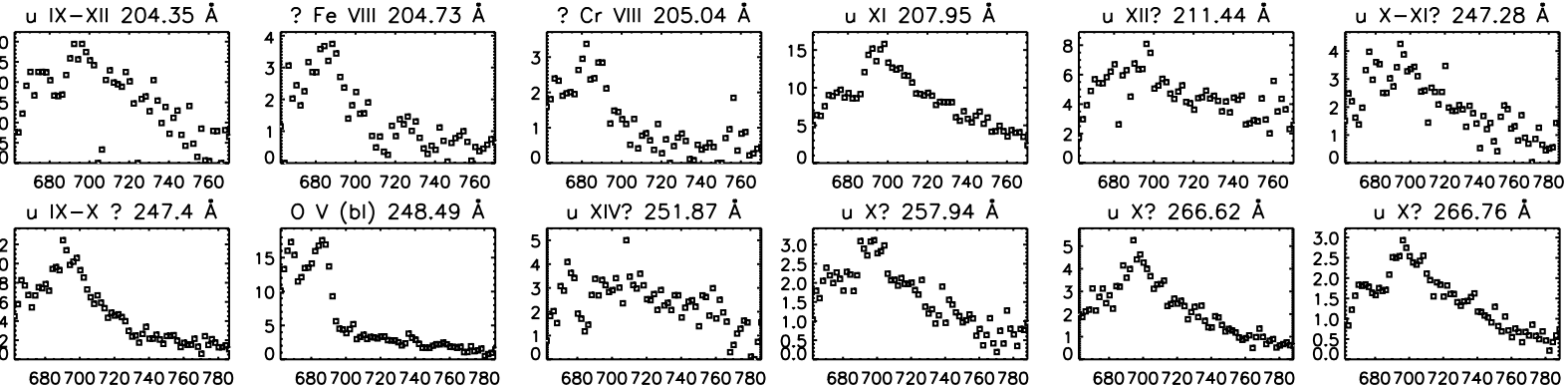

Fig. A.2. Intensities (ordinate) in a selection of Hinode/EIS lines as they vary across the solar limb (abscissa: in arcseconds along the N-S direction from disk centre, see Fig. 4). The top row shows strong well-known lines for reference, the rest are unidentified. 


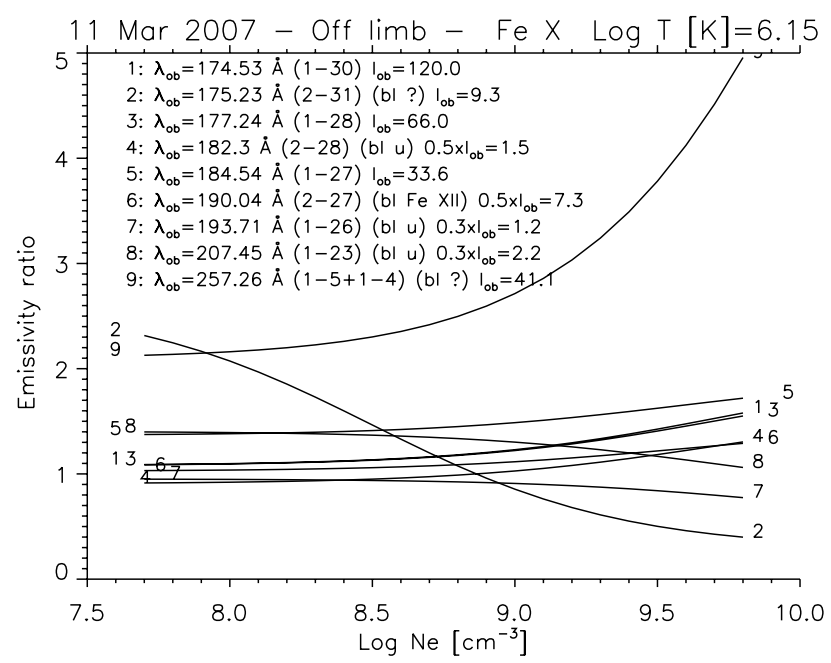

Fig. A.3. Emissivity ratio curves relative to the main Fe x EUV transitions observed by Hinode EIS on 2007 Mar. 11. $I_{\mathrm{ob}}$ : observed intensity; bl: blend; sbl: self-blend; $\mathrm{N}$ : new identification proposed here.

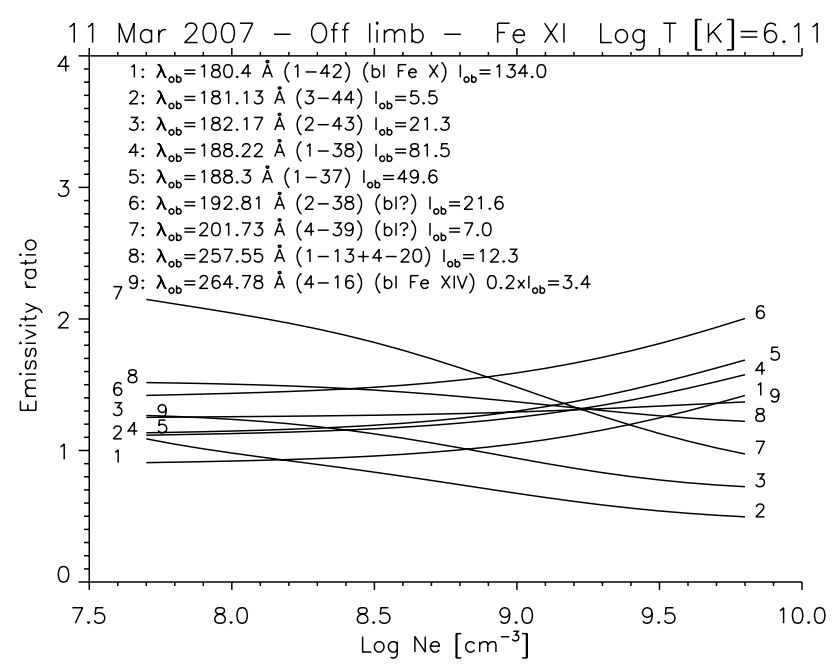

Fig. A.4. Emissivity ratio curves relative to the main Fe xi EUV transitions observed by Hinode EIS on 2007 Mar. 11.

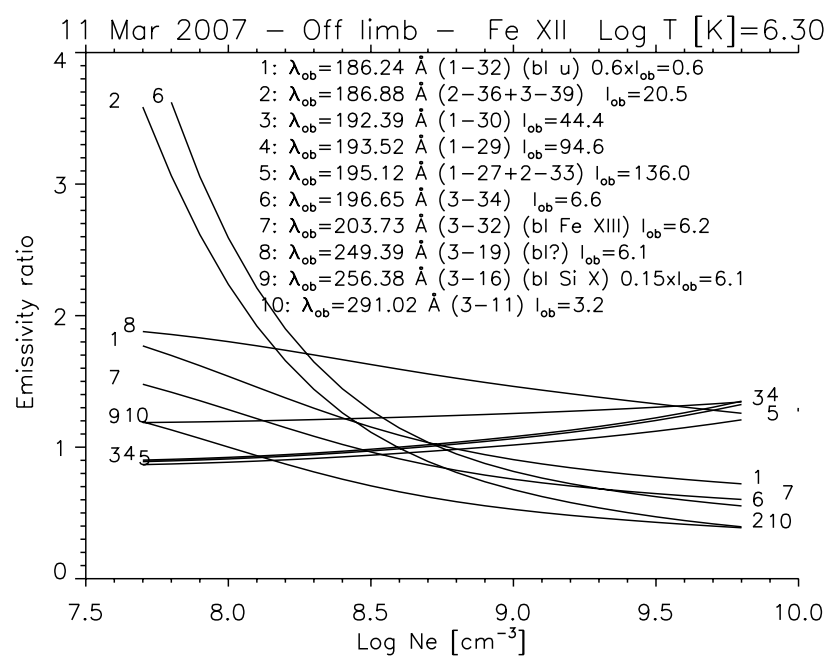

Fig. A.5. Emissivity ratio curves relative to the main Fe XII EUV transitions observed by Hinode EIS on 2007 Mar. 11. 
G. Del Zanna: Benchmarking atomic data for the CHIANTI atomic database: coronal lines observed by Hinode EIS
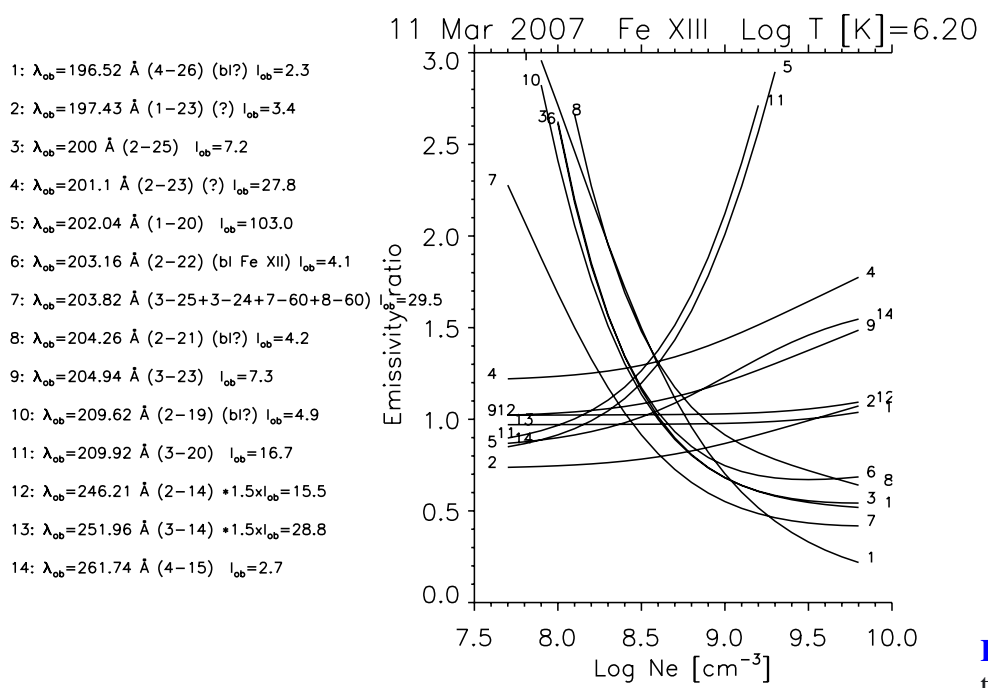

Fig. A.6. Emissivity ratio curves relative to the main Fe xIII EUV transitions observed by Hinode EIS on 2007 Mar. 11.
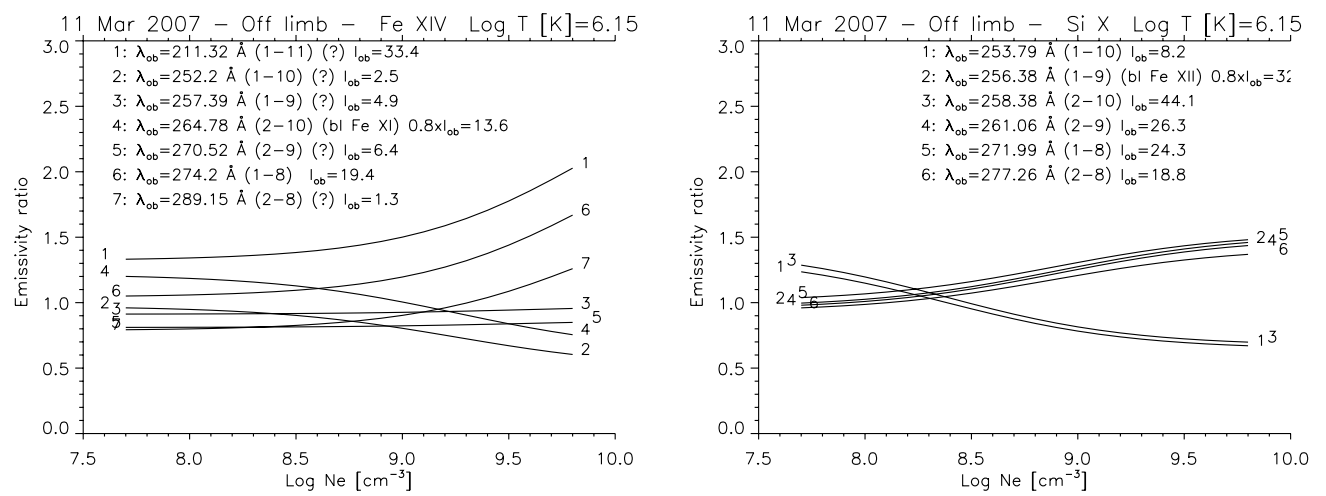

Fig. A.7. Emissivity ratio curves relative to the Fe xIV and Si x EUV transitions observed by Hinode EIS on 2007 Mar. 11. 